\title{
Front Matter: Volume 7336
}

, "Front Matter: Volume 7336," Proc. SPIE 7336, Signal Processing, Sensor Fusion, and Target Recognition XVIII, 733601 (22 May 2009); doi:

$10.1117 / 12.834334$

SPIE Event: SPIE Defense, Security, and Sensing, 2009, Orlando, Florida, United SPIE. States 


\section{PROCEEDINGS OF SPIE}

\section{Signal Processing, Sensor Fusion, and Target Recognition XVIII}

Ivan Kadar

Editor

13-15 April 2009

Orlando, Florida, United States

Sponsored and Published by

SPIE

Volume 7336

Proceedings of SPIE, 0277-786X, v. 7336 
The papers included in this volume were part of the technical conference cited on the cover and title page. Papers were selected and subject to review by the editors and conference program committee. Some conference presentations may not be available for publication. The papers published in these proceedings reflect the work and thoughts of the authors and are published herein as submitted. The publisher is not responsible for the validity of the information or for any outcomes resulting from reliance thereon.

Please use the following format to cite material from this book:

Author(s), "Title of Paper," in Signal Processing, Sensor Fusion, and Target Recognition XVIII, edited by Ivan Kadar, Proceedings of SPIE Vol. 7336 (SPIE, Bellingham, WA, 2009) Article CID Number.

ISSN 0277-786X

ISBN 9780819476029

Published by

SPIE

P.O. Box 10, Bellingham, Washington 98227-0010 USA

Telephone +1 3606763290 (Pacific Time) · Fax +1 3606471445

SPIE.org

Copyright (C) 2009, Society of Photo-Optical Instrumentation Engineers

Copying of material in this book for internal or personal use, or for the internal or personal use of specific clients, beyond the fair use provisions granted by the U.S. Copyright Law is authorized by SPIE subject to payment of copying fees. The Transactional Reporting Service base fee for this volume is $\$ 18.00$ per article (or portion thereof), which should be paid directly to the Copyright Clearance Center (CCC), 222 Rosewood Drive, Danvers, MA 01923. Payment may also be made electronically through CCC Online at copyright.com. Other copying for republication, resale, advertising or promotion, or any form of systematic or multiple reproduction of any material in this book is prohibited except with permission in writing from the publisher. The CCC fee code is 0277-786X/09/\$18.00.

Printed in the United States of America.

Publication of record for individual papers is online in the SPIE Digital Library.

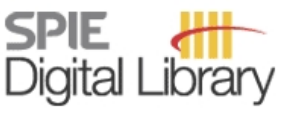

SPIEDigitalLibrary.org

Paper Numbering: Proceedings of SPIE follow an e-First publication model, with papers published first online and then in print and on CD-ROM. Papers are published as they are submitted and meet publication criteria. A unique, consistent, permanent citation identifier (CID) number is assigned to each article at the time of the first publication. Utilization of CIDs allows articles to be fully citable as soon they are published online, and connects the same identifier to all online, print, and electronic versions of the publication. SPIE uses a six-digit CID article numbering system in which:

- The first four digits correspond to the SPIE volume number.

- The last two digits indicate publication order within the volume using a Base 36 numbering system employing both numerals and letters. These two-number sets start with 00, 01, 02, 03, 04 , $05,06,07,08,09,0 A, 0 B \ldots$. OZ, followed by 10-1Z, 20-2Z, etc.

The CID number appears on each page of the manuscript. The complete citation is used on the first page, and an abbreviated version on subsequent pages. Numbers in the index correspond to the last two digits of the six-digit CID number. 


\section{Contents}

ix Conference Committee

xii Invited Panel Discussion: Issues and Challenges in: (1) Robust Methods in Tracking, Fusion and Decision Making, and (2) Sensor Bias Estimation and Data Fusion with Applications to Real-World Problems

I. Kadar, Interlink Systems Sciences, Inc. (United States); F. Daum, Raytheon Co. (United States); T. Kirubarajan, McMaster Univ. (Canada); R. P. S. Mahler, Lockheed Martin MST2 Tactical Systems (United States)

\section{SESSION 1 MULTISENSOR FUSION, MULTITARGET TRACKING, AND RESOURCE MANAGEMENT I}

733602 Gradient estimation for particle flow induced by log-homotopy for nonlinear filters [7336-01]

F. Daum, J. Huang, A. J. Noushin, M. Krichman, Raytheon Co. (United States)

$733603 \quad$ Nonlinear filters with particle flow induced by log-homotopy [7336-02]

F. Daum, J. Huang, Raytheon Co. (United States)

733604 Improved target tracking using kinematic measurements and target orientation information [7336-03]

S. Sutharsan, R. Tharmarasa, T. Kirubarajan, McMaster Univ. (Canada)

733605 Effects of measurement unobservability on neural extended Kalman filter tracking [7336-04] S. C. Stubberud, Oakridge Technology (United States); K. A. Kramer, Univ. of San Diego (United States)

733606 Post-update compensation (PUC) with maneuver indicator [7336-05]

C. Yang, Sigtem Technology, Inc. (United States); E. Blasch, Air Force Research Lab. (United States); I. Kadar, Interlink Systems Sciences, Inc. (United States)

733607 Generating reliable quality of information (Qol) metrics for target tracking [7336-07]

C. H. J. Tan, Imperial College London (United Kingdom) and DSO National Labs.

(Singapore); D. F. Gillies, Imperial College London (United Kingdom)

\section{SESSION 2 MULTISENSOR FUSION, MULTITARGET TRACKING, AND RESOURCE MANAGEMENT II}

733608 A model for graceful degradation of tracking systems [7336-08]

T. Jorgensen, J. R. McCombs, SPARTA, Inc. (United States)

733609 Automated metrics assessment system for track fusion [7336-09] W. J. Farrell III, Adaptive Methods, Inc. (United States); C. L. McCullough, R. Canavan, The Univ. of Tennessee at Chattanooga (United States) 
7336 OA Adaptive sensing for target tracking in covert operations [7336-10]

P. R. Barbosa, Colorado State Univ. (United States) and Intermap Technologies Inc. (United States); E. K. P. Chong, Colorado State Univ. (United States)

$7336 \mathrm{OB}$ A dynamic path planning algorithm for UAV tracking [7336-11]

H. Chen, K. C. Chang, George Mason Univ. (United States); C. S. Agate, Toyon Research Corp. (United States)

$73360 \mathrm{C}$ Adaptive filtering for single target tracking [7336-12]

M. Scalzo, G. Horvath, E. Jones, A. Bubalo, M. Alford, Air Force Research Lab. (United States);

R. Niu, P. K. Varshney, Syracuse Univ. (United States)

\section{SESSION 3 MULTISENSOR FUSION METHODOLOGIES AND APPLICATIONS I}

7336 OD The multisensor PHD filter: II. Erroneous solution via Poisson magic [7336-13]

R. Mahler, Lockheed Martin MS2 Tactical Systems (United States)

7336 OE The multisensor PHD filter: I. General solution via multitarget calculus [7336-14]

R. Mahler, Lockheed Martin MS2 Tactical Systems (United States)

7336 OF Maneuvering target tracking using probability hypothesis density smoothing [7336-15]

N. Nadarajah, T. Kirubarajan, McMaster Univ. (Canada)

7336 OG Joint target-detection and tracking smoothers [7336-16]

D. Clark, Heriot-Watt Univ. (United Kingdom)

\section{SESSION 4 MULTISENSOR FUSION METHODOLOGIES AND APPLICATIONS II}

$7336 \mathrm{OH}$ Sensor management of space-based multiplatform EO/IR sensors for tracking geosynchronous satellites [7336-17]

A. El-Fallah, A. Zatezalo, Scientific Systems Co., Inc. (United States); R. Mahler, Lockheed Martin Tactical Defense Systems (United States); R. K. Mehra, Scientific Systems Co., Inc. (United States); J. Brown, Air Force Research Lab. (United States)

7336 ol Dispersed and disparate sensor management for tracking low earth orbit satellites [7336-18] A. Zatezalo, A. El-Fallah, Scientific Systems Co., Inc. (United States); R. Mahler, Lockheed Martin Tactical Defense Systems (United States); R. K. Mehra, Scientific Systems Co., Inc. (United States); J. Brown, Air Force Research Lab. (United States)

7336 0J First-principles mapping of fusion applications into the JDL model [7336-19]

R. T. Antony, SAIC (United States); J. A. Karakowski, U.S. Army RDEC (United States)

7336 OK Bounds on the ROC curves from fused correlated ATR systems [7336-21]

C. M. Schubert, Virginia Commonwealth Univ. (United States); M. E. Oxley, K. W. Baver, Jr., Air Force Institute of Technology (United States)

7336 OL ROC manifolds of multiple fused independent ATR systems [7336-22]

M. E. Oxley, Air Force Institute of Technology (United States); C. M. Schubert, Virginia Commonwealth Univ. (United States); S. N. Thorsen, U.S. Air Force Academy (United States) 
7336 OM Receding horizon controller using particle swarm optimization for closed-loop ground target surveillance and tracking [7336-23]

A. J. Newman, S. R. Martin, J. T. DeSena, J. C. Clarke, J. W. McDerment, W. O. Preissler,

C. K. Peterson, The Johns Hopkins Univ. Applied Physics Lab. (United States)

7336 ON Detecting targets hidden in random forests [7336-24]

M. A. Kouritzin, D. Luo, F. Newton, B. Wu, Univ. of Alberta (Canada)

SESSION 5 MULTISENSOR FUSION METHODOLOGIES AND APPLICATIONS III

733600 Performance analysis of structured pedigree distributed fusion systems [7336-25]

P. O. Arambel, BAE Systems (United States)

7336 OP An epidemic model for biological data fusion in ad hoc sensor networks [7336-26]

K. C. Chang, V. Kotari, George Mason Univ. (United States)

SESSION 6 MULTISENSOR FUSION METHODOLOGIES AND APPLICATIONS IV

$73360 Q \quad$ An AOA-based optimal sensor locations' self-adjusting with moving targets' location estimation [7336-28]

J. Luo, E. Walker, P. Bhattacharya, X. Chen, Southern Univ. and A\&M College (United States)

7336 OR A near-optimal low complexity sensor fusion technique for accurate indoor localization based on ultrasound time of arrival measurements from low-quality sensors [7336-29]

S. A. Mitilineos, N. D. Argyreas, S. C. A. Thomopoulos, National Ctr. for Scientific Research Demokritos (Greece)

7336 OS Analysis of sniper localization for mobile asynchronous sensors [7336-30]

G. T. Whipps, L. M. Kaplan, R. Damarla, Army Research Lab. (United States)

\section{SESSION 7 MULTISENSOR FUSION METHODOLOGIES AND APPLICATIONS V}

7336 OT A risk-based comparison of classification systems [7336-31]

S. Thorsen, U.S. Air Force Academy (United States); S. Wagenman, M. E. Oxley, K. W. Baver, Air Force Institute of Technology (United States)

$7336 \mathrm{OU}$ The effects of correlation on the performance of ATR systems [7336-32]

C. M. Schubert, Virginia Commonwealth Univ. (United States); S. N. Thorsen, U.S. Air Force Academy (United States); M. E. Oxley, Air Force Institute of Technology (United States)

7336 OV The exact fundamental solution for the Beňes tracking problem [7336-33]

B. Balaji, Defence Research and Development Canada, Ottawa (Canada)

7336 OW Efficient target tracking using adaptive grid and sparse tensors [7336-34]

B. Balaji, Defence Research and Development Canada, Ottawa (Canada) 
7336 OX Object recognition methodology for the assessment of multi-spectral fusion algorithms: phase 1 [7336-35]

A. R. Pinkus, Air Force Research Lab. (United States); A. Toet, TNO Defense, Security and Safety (Netherlands); H. L. Task, Task Consulting (United States)

7336 OY Multi-sensor management for data fusion in target tracking [7336-36]

X. Li, G. Chen, DCM Research Resources, LLC (United States); E. Blasch, J. Patrick, Air Force Research Lab. (United States); C. Yang, Sigtem Technology, Inc. (United States); I. Kadar, Interlink Systems Sciences, Inc (United States)

$7336 \mathrm{OZ} \quad$ Fusion of multi-sensory saliency maps for automated perception and control [7336-37] D. J. Huber, D. Khosla, P. A. Dow, HRL Labs., LLC (United States)

SESSION 9 SIGNAL AND IMAGE PROCESSING I

733612 Dynamic algorithm selection for multi-sensor image registration [7336-40]

S. DelMarco, V. Tom, H. Webb, D. Lefebvre, BAE Systems Inc. (United States)

733613 Shadow and feature recognition aids for rapid image geo-registration in UAV vision system architectures [7336-41]

W. Baer, M. Kölsch, Naval Postgraduate School (United States)

733614 Image-based querying of urban knowledge databases [7336-42]

P. Cho, MIT Lincoln Lab. (United States); S. Bae, F. Durand, MIT Computer Science \& Artificial Intelligence Lab. (United States)

733615 CASSIE: contextual analysis for spectral and spatial information extraction [7336-43]

L. Gibson, J. Horne, D. Haverkamp, SAIC (United States)

733617 High-resolution reconstruction of objects from cloud-covered infrared images [7336-45]

J. Wang, J. F. Ralph, J. Y. Goulermas, The Univ. of Liverpool (United Kingdom)

733619 Rural road extraction from SPOT images based on a Hermite transform pansharpening fusion algorithm [7336-47]

B. Escalante-Ramírez, Univ. Nacional Autónoma de México (Mexico) and Ctr. de Investigación en Geografía y Geomática (Mexico); A. A. López-Caloca, Ctr. de Investigación en Geografía y Geomática (Mexico)

7336 1A Development of performance metrics to characterize the degree of polarization of man-made objects using passive polarimetric images [7336-48]

D. A. Lavigne, Defence Research and Development Canada, Valcartier (Canada); M. Breton, AEREX Avionics inc. (Canada); G. Fournier, M. Pichette, Defence Research and Development Canada, Valcartier (Canada); V. Rivet, AEREX Avionics inc. (Canada)

7336 1B Man-made objects cuing in satellite imagery [7336-49]

A. N. Skurikhin, Los Alamos National Lab. (United States)

7336 1C Quad-emissive display for multi-spectral sensor analyses [7336-50]

A. R. Pinkus, Air Force Research Lab. (United States); H. L. Task, Task Consulting (United States) 
7336 1D An optimal nonlinear filter for detecting non-normality in a signal using the bicoherence [7336-51]

J. M. Nichols, C. Olson, Naval Research Lab. (United States); J. Michalowicz, Global

Strategies Group (United States); F. Bucholtz, Naval Research Lab. (United States)

$7336 \mathrm{IF}$ Use of unidirectional microphones and signal processing for the localization of sound sources [7336-53]

P. Rizzo, Univ. of Pittsburgh (United States); G. Bordoni, Italcementi Group (Italy); A. Marzani, Univ. of Bologna (Italy)

7336 IG Statistical modeling of interferometric signals in underwater applications [7336-54]

G. Llort, C. Sintes, Intitut Télécom-Télécom Bretagne, CNRS, Lab.-STICC (France)

$7336 \mathrm{1H} \quad$ Tracking dust: tracking and state estimation for dusty plasmas [7336-55]

J. F. Ralph, D. Samsonov, C. Durniak, The Univ. of Liverpool (United Kingdom); G. E. Morfill, Max-Planck-Institut für Extraterrestrische Physik (Germany)

\section{SESSION 11 SIGNAL AND IMAGE PROCESSING III}

$733611 \quad$ Perimeter intrusion detection and intruder classification [7336-56]

J. F. Smith III, Naval Research Lab. (United States)

7336 1J Detecting modulated lasers in the battlefield and determining their direction [7336-57]

A. D. McAulay, Lehigh Univ. (United States)

7336 IK Decomposing radar measurements through comprehensive response modeling [7336-58] I. Venäläinen, J. Jylhä, V. Väisänen, J. Vihonen, M. Ruotsalainen, A. Visa, Tampere Univ. of Technology (Finland)

\section{POSTER SESSION}

733610 Multispectral air pollution sensor to characterize the total suspended particulates (TSP) concentration [7336-65]

S. G. Daraigan, Univ. Sains Malaysia (Malaysia) and Hadhramout Univ. of Science and Technology (Yemen); M. Z. MatJafri, K. Abdullah, H. S. Lim, C. J. Wong, Univ. Sains Malaysia (Malaysia)

7336 IP Temporal and spatial air quality monitoring using internet protocol camera and LANSAT5 satellite image [7336-66]

C. J. Wong, H. S. Lim, M. Z. MatJafri, K. Abdullah, T. S. Chin, Univ. Sains Malaysia (Malaysia)

Author Index 
Downloaded From: https://www.spiedigitallibrary.org/conference-proceedings-of-spie on 26 Apr 2023

Terms of Use: https://www.spiedigitallibrary.org/terms-of-use 


\title{
Conference Committee
}

\author{
Symposium Chair
}

Ray O. Johnson, Lockheed Martin Corporation (United States)

Symposium Cochair

Michael T. Eismann, Air Force Research Laboratory (United States)

Conference Chair

Ivan Kadar, Interlink Systems Sciences, Inc. (United States)

Program Committee

Mark G. Alford, Air Force Research Laboratory (United States)

William D. Blair, Georgia Tech Research Institute (United States)

Erik P. Blasch, Air Force Research Laboratory (United States)

Mark J. Carlotto, General Dynamics Advanced Information Systems (United States)

Kuo-Chu Chang, George Mason University (United States)

Chee-Yee Chong, BAE Systems Advanced Information Technologies (United States)

Marvin N. Cohen, Georgia Tech Research Institute (United States)

Mohammad Farooq, Royal Military College of Canada (Canada)

Charles W. Glover, Oak Ridge National Laboratory (United States)

I. R. Goodman, Consultant (United States)

Lynne L. Grewe, California State University, East Bay (United States)

Michael L. Hinman, Air Force Research Laboratory (United States)

Kenneth J. Hintz, George Mason University (United States)

Jon S. Jones, Air Force Research Laboratory (United States)

Thiagalingam Kirubarajan, McMaster University (Canada)

Martin E. Liggins II, MITRE Corporation (United States)

James Llinas, University at Buffalo (United States)

Ronald P. Mahler, Lockheed Martin Corporation (United States)

Raj P. Malhotra, Air Force Research Laboratory (United States)

Alastair D. McAulay, Lehigh University (United States)

Raman K. Mehra, Scientific Systems Company, Inc. (United States)

Harley R. Myler, Lamar University (United States)

David Nicholson, BAE Systems plc (United Kingdom)

Leslie M. Novak, BAE Systems Advanced Information Technologies (United States)

Andrew G. Tescher, AGT Associates (United States) 
Stelios C. A. Thomopoulos, National Center for Scientific Research

Demokritos (Greece)

Wiley E. Thompson, New Mexico State University (United States)

Pierre Valin, Defence R\&D Canada, Valcartier (Canada)

\section{Session Chairs}

1 Multisensor Fusion, Multitarget Tracking, and Resource Management I

Ivan Kadar, Interlink Systems Sciences, Inc. (United States)

Thiagalingam Kirubarajan, McMaster University (Canada)

2 Multisensor Fusion, Multitarget Tracking, and Resource Management II

Ivan Kadar, Interlink Systems Sciences, Inc. (United States)

Kenneth J. Hintz, George Mason University (United States)

3 Multisensor Fusion Methodologies and Applications I

Ronald P. Mahler, Lockheed Martin Corporation (United States)

4 Multisensor Fusion Methodologies and Applications II

Ronald P. Mahler, Lockheed Martin Corporation (United States)

5 Multisensor Fusion Methodologies and Applications III

Michael L. Hinman, Air Force Research Laboratory (United States)

Kenneth J. Hintz, George Mason University (United States)

Ivan Kadar, Interlink Systems Sciences, Inc. (United States)

6 Multisensor Fusion Methodologies and Applications IV

Kenneth J. Hintz, George Mason University (United States)

Ivan Kadar, Interlink Systems Sciences, Inc. (United States)

Michael L. Hinman, Air Force Research Laboratory (United States)

7 Multisensor Fusion Methodologies and Applications $V$

Ivan Kadar, Interlink Systems Sciences, Inc. (United States)

Michael L. Hinman, Air Force Research Laboratory (United States)

Kenneth J. Hintz, George Mason University (United States)

$8 \quad$ Multisensor Fusion Methodologies and Applications VI

Michael L. Hinman, Air Force Research Laboratory (United States)

Kenneth J. Hintz, George Mason University (United States)

Ivan Kadar, Interlink Systems Sciences, Inc. (United States)

$9 \quad$ Signal and Image Processing I

Lynne L. Grewe, California State University, East Bay (United States)

Alastair D. McAulay, Lehigh University (United States)

Mark G. Alford, Air Force Research Laboratory (United States) 
10 Signal and Image Processing II

Mark G. Alford, Air Force Research Laboratory (United States)

Alastair D. McAulay, Lehigh University (United States)

Lynne L. Grewe, California State University, East Bay (United States)

11 Signal and Image Processing III

Alastair D. McAulay, Lehigh University (United States)

Lynne L. Grewe, California State University, East Bay (United States)

Mark G. Alford, Air Force Research Laboratory (United States) 
Downloaded From: https://www.spiedigitallibrary.org/conference-proceedings-of-spie on 26 Apr 2023

Terms of Use: https://www.spiedigitallibrary.org/terms-of-use 


\section{Invited Panel Discussion}

Issues and Challenges in: (1) Robust Methods in Tracking, Fusion, and Decision Making, and (2) Sensor Bias Estimation and Data Fusion with Applications to Real-World Problems

\section{Organizer}

Ivan Kadar, Interlink Systems Sciences, Inc.

\section{Moderators}

Ivan Kadar, Interlink Systems Sciences, Inc.

Frederick E. Daum, Raytheon Company

April 13, 2009

SPIE Conference 7336

"Signal Processing, Sensor Fusion and Target Recognition XVIII" Orlando, FL April 13-15, 2008

\section{Invited Panel Discussion}

\section{Participants:}

${ }^{*}$ Dr. Dale Blair, Georgia Tech. Research Institute, U.S.A.

${ }^{*}$ Dr. Erik P. Blasch, Air Force Research Lab., U.S.A.

${ }^{*}$ Dr. Chee-Yee Chong, BAE Systems, U.S.A.

Dr.Frederick E. Daum, Raytheon Co., U.S.A.

Dr. Ivan Kadar, Interlink Systems Sciences, Inc., U.S.A.

Professor Thiagalingam Kirubarajan, McMaster Univ.(Canada)

Dr. Ronald P. S. Mahler, Lockheed Martin MST2 Tactical Systems, U.S.A.

* Unable to attend 


\section{Invited Panel Discussion}

\section{Topics}

" Is there a Royal Road to Robustness?"

Dr. Frederick E. Daum, Raytheon Co.

"On Robust Estimation: Methods, Applications and Challenges"

Dr. Ivan Kadar, Interlink Systems Sciences, Inc.

"Robustness, Unknowability, and Joe Btfsplk"

Dr. Ronald P. S. Mahler, Lockheed Martin MS2 Tactical

Systems

"Tracking with Transmitter of Opportunity: A Real-World Problem with Bias, Clutter and Fusion"

Professor Thiagalingam Kirubarajan, McMaster Univ. (Canada)

“ An Impromptu Presentation: Real-World Sensor Bias Effects and Challenges"

Dr. Frederick E. Daum, Raytheon Co. 


\title{
Is there a Royal Road to Robustness?
}

\author{
Fred Daum
}

There is much confusion and misinformation about robustness among engineers. For example, many smart hard working and well educated engineers believe that there are decision rules and estimation algorithms that are more robust than Bayesian algorithms. In particular, many engineers think that fuzzy logic or Dempster-Shafer methods are more robust than Bayesian methods. In this paper, to be specific, we will consider one specific paper written by an engineer [8].

Joseph Carl has written a long and provocative chapter [8] on Bayesian vs. Dempster-Shafer (D-S) decision algorithms, which have been the subject of heated debates over the last several decades. Carl says that "many would argue that probability theory is not suitable for practical implementation on complex real-world problems," which is a very interesting assertion, but Carl does not explain how D-S theory improves the situation. In particular, it is well known and not subject to debate that given a decision problem with completely defined probability distribution functions (pdfs), that Bayesian decision rules are optimal. Therefore, the only way in which D-S could improve performance is in the case in which the pdfs are not completely specified. The problem of incomplete pdfs was the original motivation for Professor Dempster's seminal work published in 1967 and 1968, and it has been the subject of extensive research since then, but without any practical results so far (see Walley's book [1] and pages 57-61 in [2] for a survey of this research). The best work that $I$ know of on this subject is chapter 5 of Kharin's recent book [3], which basically concludes that for most sensible models of uncertainty in pdfs, the standard Bayesian decision rule, or a minor modification of it, is the most robust approach. There are a number of interesting mathematical questions in Kharin's book, such as: why is the Bayesian decision rule robust relative to perturbations of the pdf in L1 but not in L2? One possible answer is that probability densities live in $L 1$ rather than $\mathrm{L} 2$, and thus $\mathrm{L1}$ is a good space in which to measure perturbations to pdfs, whereas $L 2$ is not. That is, allowing $L 2$ perturbations to pdfs that do not live in $\mathbf{L} 2$ can result in perturbations that are not densities at all. Moreover, Scheffé's machinery shows that $L 1$ is the appropriate space in which to measure errors in probability densities (see Luc Devroye's books [12]-[13] for 
more details, especially page 52 of [12]). But this question deserves deeper analysis elsewhere. There is no formula or theorem in Carl's chapter that shows how much D-S improves performance relative to Bayesian methods, nor does Carl assert that D-S is better than Bayes, but rather in the detailed numerical example that is worked out, the $D-S$ and Bayesian decisions are "qualitatively the same." This agrees with other comparisons of D-S with Bayes reported in the literature, when such calculations are done correctly. There are, unfortunately, notorious cases of papers published by engineers in which the D-S advocate claimed superiority over Bayes, owing to the engineer's faulty understanding of elementary probability theory. Moreover, it is well known that Dempster's rule of combining evidence assumes that the data are statistically independent, whereas Bayesian methods do not require this assumption, with the result that for problems with statistically dependent data, the Bayesian performance can be vastly superior to D-S. This is a fundamental limitation of D-S compared with Bayes, but Carl does not mention it in his chapter, which is a curious lacuna in a handbook on data fusion such as this. A surprisingly common misconception is that Bayesian methods require Gaussian pdfs, uniform prior distributions and/or statistically independent data, whereas in fact Bayesian decision rules are optimal for any pdf of the data (Gaussian or non-Gaussian), any prior pdf (uniform or non-uniform) and data that are statistically dependent or independent. Three good references on D-S vs. Bayesian methods, which are both readable by normal people as well as being mathematically correct are: Walley's book [1], chapter 9 of Pearl's book [6] and Wasserman's extremely lucid paper [7]. Carl mentions near the end of his chapter that D-S "is not equipped with a clear-cut decision making rule," which many readers will find somewhat surprising, because $D-S$ has been touted as a better method of decision making. For those wondering what it means to say that D-S has no decision rules, one should read chapters 13 to 17 in [4]. Moreover, in the foreword to Shafer's book [5], Professor Dempster very clearly says that: "I differ from Shafer....I believe that Bayesian inference will always be a basic tool for practical everyday statistics, if only because questions must be answered and decisions must be taken." So Professor Dempster is apparently a Bayesian, and he would not advocate the use of D-S for decision making. Many years ago, mathematicians gave up trying to make better decisions than Bayes; the recent papers on D-S say that there is more to life than making decisions, that Bayes wins at making decisions, and that maybe D-S is useful to "represent" uncertainty. However, hard-boiled engineers are only interested in making decisions; "representing uncertainty" is for poets, not engineers. 
A few years ago a day long workshop was organized by MIT Lincoln Lab on the subject of Bayes vs. Dempster-Shafer algorithms. The meeting was very well attended by both D-S researchers and Bayesians, including Professor Dempster and Professor Smets. The specific purpose of this meeting was to get to the bottom of the situation; is D-S better than Bayes or not? At this meeting, nobody asserted that D-S was any better than Bayes, perhaps out of fear of being cut to ribbons in front of their sponsor.

One could analyze the similar situation that obtains for fuzzy logics vs. Bayesian decision rules, but Professor Lindley has already done this, in a remarkably short paper [10]. I have expounded at length elsewhere on fuzzy sets vs. Bayesian algorithms [11].

In summary, there is no royal road to robustness. In particular, there is no decision rule or estimation algorithm that will improve robustness relative to the optimal Bayesian algorithm. People who assert that fuzzy logic or Dempster-Shafer are more robust than Bayesian algorithms are selling snake oil. Moreover, Kharin [3] has proved that the standard Bayesian decision rule is optimally robust with respect to pertubations in $L 1$. Rather than wasting their time searching for the mythical royal road to robustness, engineers can increase robustness by using many good methods, including: (1) add margin to the design, (2) use feedback, (3) collect more relevant data to reduce uncertainty, (4) use physics, (5) use more physics, (6) model uncertainty in the sensors, targets and environment, (7) use adaptive algorithms to estimate the statistics of clutter \& jamming \& target maneuvers (e.g., CFAR, IMM, clutter maps), (8) use Tychonoff regularization (or any of a dozen other methods) to mitigate ill-conditioning, (9) use the minimum number of states to accurately model the relevant physical situation, (10) use good data association algorithms (e.g., MHT, JVC, JPDA, PDA), (11) model unresolved measurements, (12) model residual sensor bias, (13) understand the physics of sensor bias (e.g., tropospheric refraction, ionospheric refraction, IMU calibration, radome refraction, antenna or focal plane array mechanical $\&$ electrical \& optical bias, thermally induced errors in antenna \& IMU, ionospheric \& multipath errors in GPS, etc.), (14) use a Bayesian decision rule, which is inherently robust, (15) use better sensors or more sensors, and (16) for multisensor data fusion in a dense multiple target environment, use an algorithm that jointly estimates residual sensor bias and estimates data association (e.g., GNPL). 


\section{REFERENCES}

[1] Walley, P. "Statistical Reasoning with Imprecise Probabilities," Chapman and Hall, 1991.

[2] "Robust Bayesian Analysis," edited by D. Insua and F. Ruggeri, SpringerVerlag, 2000.

[3] Kharin, Y., "Robustness in Statistical Pattern Recognition," Kluwer, 1996.

[4] "Advances in the Dempster-Shafer Theory of Evidence," edited by R.R. Yager, et al., John Wiley \& Sons, 1994.

[5] Shafer, G., "A Mathematical Theory of Evidence," Princeton University Press, 1976.

[6] Pearl, J., "Probabilistic Reasoning in Intelligent Systems," Morgan Kaufmann, 1988.

[7] Wasserman, L., "Belief functions and statistical inference," Canadian Journal of Statistics, 1990.

[8] Joseph Carl, "Dempster-Shafer vs. Bayesian methods," Chapter 7 in "Handbook of Multisensor Data Fusion," edited by David Hall and James Llinas, CRC Press, 2001.

[9] "Fuzziness vs. Probability -- $\mathbf{N}^{\text {th }}$ Round", Special issue of IEEE Transactions on Fuzzy Systems, Feb. 1994.

[10] D. V. Lindley, "Comments on 'The Efficacy of Fuzzy Representations of Uncertainty"", op. cit. p.37.

[11] Fred Daum, "Cramér-Rao Type Bounds for Random Set Problems," Chapter 8 in "Random Sets: Theory and Applications," edited by J. Goutsias, R. Mahler and H. T. Nguyen, Springer-Verlag, 1997.

[12] Luc Devroye \& Gábor Lugosi, "Combinatorial methods in density estimation," Springer-Verlag, 2001. 
[13] Luc Devroye \& L. Györfi, "Nonparametric density estimation: the L1 view," John Wiley \& Sons, 1985.

[14] Carl F. Gauss, "Unstinken methode der kleinsten quadrate," Werke, Königlichen Gesellschaft der Wissenschaften zu Göttingen, 1870-1928.

[15] Peter Huber, "Robust Statistical Procedures," $2^{\text {nd }}$ edition, SIAM (1996).

[16] Peter Huber, “Robust Statistics,” John Wiley \& Sons, 1981.

[17] George Box, "Sampling and Bayes' inference in scientific modelling and robustness" with discussion and rejoinder, Journal of Royal Statistical Society, 1980. 


\title{
Is there a Royal Road to Robustness?
}

\author{
Fred Daum
}

\section{April 2009}

\section{Raytheon}

\section{Examples of methods to increase robustness}

- Add margin to the design (e.g., "safety factor" in building a bridge)

- Feedback

- CFAR

- Clutter Map

- Adaptive Nulling

- STAP

- Adaptive Kalman Filters, IMM, PDA, JVC, JPDA, MHT, GNPL, etc.

- Tune process noise of EKF

- Decouple covariance matrix of EKF

- Rejection of outliers

- Median vs. mean

- Huber estimators

- Fatten the tails of the probability densities (e.g., MVT vs. Gaussian)

- Noninformative prior

- Occam's razor

- Reduce dimensionality

- Increase dimensionality 


\section{Examples of methods to increase robustness}

- Smoothing

- Coarsening

- Density estimation vs. textbook densities

- Robust Bayes

- Model uncertainty in the models and probability distributions

- Collect more data for off-line training of algorithms

- Collect more data on-line adaptively

- Multiple sensor fusion

- Improved sensors

- Get help from experienced people, who know what the list of important issues is

- System Spec. with explicit statistical variation quantified in all relevant parameters and models

- Use physics

- Use more physics

- Other

\section{Raytheon}

\section{Theoretical Literature}

1. Peter Huber, Robust Statistical Procedures, second edition, SIAM (1996)

2. Peter J. Huber, Robust Statistics, John Wiley \& Sons, 1981

3. Yurij Kharin, Robustness in Statistical Pattern Recognition, Kluwer, 1996.

4. Robust Bayesian Analysis, edited by David Ros Insua, et. al., Springer (2000).

5. Bayesian Robustness, edited by J. $O$. Berger, et al., IMS, 1996.

6. Frank Hampel, et al., Robust Statistics, John Wiley \& Sons, 1986.

7. Robust Inference, edited by Maddala and Rao, North-Holland (1997).
Concise (only 67 pages) and authoritative. The final words of the master.

Methods to improve robustness for estimation using classical statistics

Bayesian analysis of robustness for decision problems, with nice explicit results, approximate formulas, for a wide range of contamination models, and optimal robust decision rules. Must read Chapter 5.

440 pages of robust Bayesianism hot off the press, but there is no mention of Kharin's work!?*

Conference proceedings on sensitivity analysis with Bayesian formalism Local robustness within Huber's framework. Much anti-Bayesian rhetoric.

698 pages of robust classical statistics. 


\section{Raytheon}

\section{Theoretical Literature}

8. Peter Walley, Statistical Reasoning with Imprecise Probabilities, Chapman \& Hall, 1991.

9. Arthur Dempster, "Upper and Lower Probabilities Induced from Multivalued Mapping," Annals of Math. Stat. 1967.

10.Larry Wasserman, "Belief functions and statistical inference," Canadian Journal of Statistics, 1990.

11.Judea Pearl, Probabilistic Reasoning in Intelligent Systems, Morgan Kaufman (1988)

12.Advances in the Dempster-Shafer Theory of Evidence, edited by Yager, et al., John Wiley \& Sons (1994)

13.Peter Walley, "TBS" (2000).

14.Larry Wasserman, "Recent Methodologica Advances in Robust Bayesian Inference," in Bayesian Statistics, edited by Nernardo, et al., Oxford University Press (1992)
Very interesting and careful analysis of attempts to go beyond Bayesian formalism

Interesting attempt to see what could be done with "incomplete" probability distributions

The most lucid introduction to DempsterShafer methods from a Bayesian viewpoint

Very readable and solid comparison of

Bayesian vs. Dempster-Shafer methods in Chapter 9.

Careful mathematical work, with surprisingly little anti-Bayesian rhetoric. Read chatpers 13 to 17 to understand what it means to say that there is no decision theory for DempsterShafer.

The latest attempt to go beyond Bayesian methods

Lucid and solid mathematics, with excellent discussions, including non-informative priors and fatter tails.

\section{Raytheon}

\section{Theoretical Literature}

\begin{tabular}{|l|l|}
\hline $\begin{array}{l}\text { 15.Robert Kass and Larry } \\
\text { Wasserman, "The Selection of } \\
\text { Prior Distribitons by Formal } \\
\text { Rules," J. Amer. Stat. Assoc., } \\
\text { (1996) }\end{array}$ & $\begin{array}{l}\text { Lucid and solid mathematics, with } \\
\text { long annotated bibliography, with } \\
\text { focus on noninformative priors and } \\
\text { other good ideas. }\end{array}$ \\
\hline $\begin{array}{l}\text { 16.James Berger, "Robust Bayesian } \\
\text { Analysis: Sensitivity to the Prior," } \\
\text { J. Stat. Planning and Inference, } \\
\text { (1990). }\end{array}$ & $\begin{array}{l}\text { Very readable and sensible, including } \\
\text { frank comments about "silly priors" }\end{array}$ \\
\hline $\begin{array}{l}\text { 17. Peter Bickel, "Another Look at } \\
\text { Robustness: A Review of } \\
\text { Reviews and Some New } \\
\text { Developments," Scand. J. Stat., } \\
\text { 1976 }\end{array}$ & $\begin{array}{l}\text { Solid mathematical survey of } \\
\text { robustness for classical statistics, } \\
\text { with stimulating discussion. }\end{array}$ \\
\hline $\begin{array}{l}\text { 18. S. Kassam and H. V. Poor, } \\
\text { "Robust Techniques for Signal } \\
\text { Processing," IEEE Proceedings, } \\
\text { 1985. }\end{array}$ & $\begin{array}{l}\text { Lucid survey of robust signal } \\
\text { processing, including robust phased } \\
\text { array antennas, robust matched } \\
\text { filters, and robust CFAR. }\end{array}$ \\
\hline $\begin{array}{l}\text { 19. Robust Control, edited by Peter } \\
\text { Dorato, IEEE Press, 1987. }\end{array}$ & $\begin{array}{l}\text { Read the introduction for a good solid } \\
\text { mathematical survey, and read the } \\
\text { papers by Doyle and Stein. }\end{array}$ \\
\hline 20. I. R. Peterson, "Robust Kalman & Solid mathematical introduction. \\
Filtering Methods," 2000. & \multicolumn{1}{c}{ SE01-097-3 } \\
\hline
\end{tabular}




\section{Raytheon}

\section{Kharin's Robust Bayesian Formulation}

$$
\mathbf{R}^{*}=\min _{\mathbf{d}}\left\{\begin{array}{l}
\max \\
\mathbf{p}_{\in} \mathbf{S}
\end{array} \mathbf{R}(\mathbf{d}, \mathbf{p})\right\}
$$

d = decision

$p=$ probability distribution

$R(d, p)=$ Bayes risk

$\mathbf{R}^{*}=$ robust Bayes risk

$S=$ admissible set of probability distributions

\section{Raytheon}

\section{Examples of Contamination Models}

\begin{tabular}{|c|c|c|c|}
\hline Model & $\begin{array}{c}\text { Is standard } \\
\text { Bayes decision } \\
\text { rule optimally } \\
\text { robust? }\end{array}$ & $\begin{array}{l}\text { Assumptions } \\
\text { for Bayes } \\
\text { decision rule } \\
\text { to be robust }\end{array}$ & $\begin{array}{c}\text { Robust } \\
\text { Bayesian } \\
\text { decision rule }\end{array}$ \\
\hline $\begin{array}{l}\text { 1. } L^{1} \text { metric: } \\
\int \mid p\left(x \mid H_{j}\right)-p_{o}\left(x \mid H_{j}\right) d x \leq \overline{\epsilon_{j}}\end{array}$ & Yes & $\operatorname{Pr}\left(H_{j} \mid H_{j}\right) \geq \bar{\epsilon}_{j}$ & $\begin{array}{c}\text { Standard } \\
\text { Bayes }\end{array}$ \\
\hline $\begin{array}{l}\text { 2. } L^{2} \text { metric: } \\
\iint p\left(x \mid H_{j}\right)-p_{o}\left(x \mid H_{j}\right)^{2} d x<\bar{\epsilon}_{j}\end{array}$ & No & - & $\begin{array}{c}\text { Nonlinear } \\
\text { mathematical } \\
\text { programming } \\
\text { with } \\
\text { constraints } \\
\end{array}$ \\
\hline $\begin{array}{l}\text { 3. Tukey-Huber: } \\
\mathbf{p}\left(\mathbf{x} \mid \mathrm{H}_{\mathrm{i}}\right)=\mathbf{p}_{\mathrm{o}}\left(\mathbf{x} \mid \mathrm{H}_{\mathrm{i}}\right)\left(\mathbf{1}-\epsilon_{\mathrm{i}}\right)+\epsilon_{\mathrm{i}} \mathbf{q}\left(\mathbf{x} \mid \mathrm{H}_{\mathrm{i}}\right)\end{array}$ & Sometimes & $\begin{array}{c}\text { Equal } \\
\text { maximum } \\
\text { contamination } \\
\text { for all } \\
\text { hypotheses } \\
\end{array}$ & $\begin{array}{c}\text { Trivial } \\
\text { modification } \\
\text { of standard } \\
\text { Bayesian } \\
\text { decision rule } \\
\end{array}$ \\
\hline $\begin{array}{l}\text { 4. Additive random variables: } \\
\qquad \begin{array}{l}z_{i}=x_{i}+\epsilon_{i} y_{i} \\
p\left(y_{i}\right)= \\
\text { unknown with given mean \& } \\
\text { covariance }\end{array}\end{array}$ & No & - & $\begin{array}{l}\text { Straight- } \\
\text { forward mod. } \\
\text { of standard } \\
\text { Bayesian } \\
\text { decision rule. }\end{array}$ \\
\hline
\end{tabular}




\section{Raytheon}

\section{Theorem for $\mathrm{L}^{1}$ Contamination Model}

If the standard Bayesian decision rule has "pretty good performance" $\left(\operatorname{Pr}\left(H_{j} \mid H_{j}\right) \geq \bar{\epsilon}_{j}\right.$ for $\left.\mathbf{j}=1,2, \ldots, L\right)$,

then it is optimally robust.

Proof: See Chapter 5 in Kharin (1996)

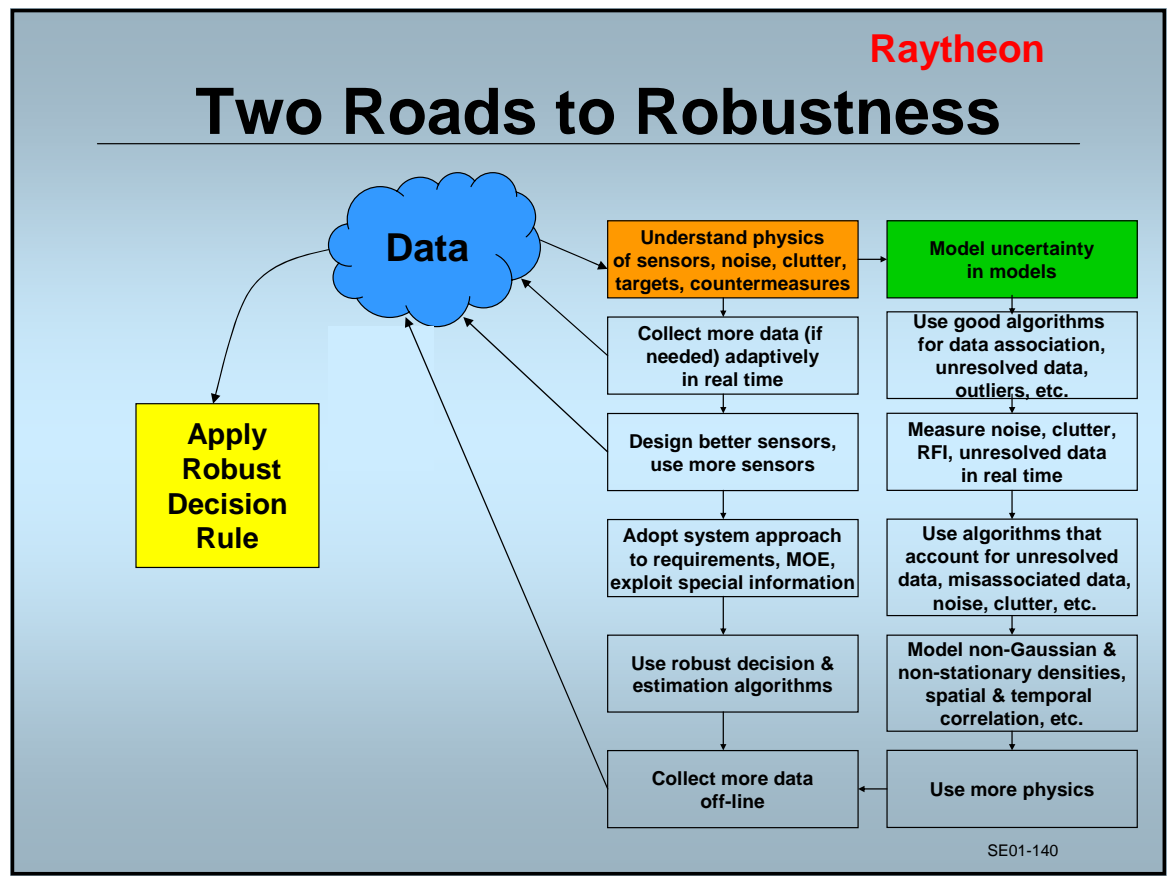




\title{
On Robust Estimation: Methods, Applications and Challenges
}

\author{
Ivan Kadar* \\ Interlink Systems Sciences, Inc., 1979 Marcus Ave., STE 210, Lake Success, NY 11042
}

\section{PERSPECTIVES ON ROBUSTNESS AND ROBUST ESTIMATION/FILTERING}

The purpose of this position paper is to complement the associated viewgraphs, provide additional insights on robustness, and to elaborate on "robust" estimation-filtering-tracking methods that are implied, but not addressed, in the associated viewgraphs, i.e., as stated: "Does a practical general robustizing method exists for the linearized Kalman Filter (KF)? Bayesian estimation methods have been shown to be intrinsically "robust" to changes in the underlying assumptions (i.e., in the face of unknown i.i.d. measurement and process noise, and nonlinearities)".

\section{Definition of Robustness and Robust Algorithms Types}

The first question one should ask is why study robustness? As addressed in accompanying viewgraphs, (1) one never has very accurate knowledge of underlying probability density function (pdf), (2) natural phenomena and man-made disturbances are rarely Gaussian, e. g., clutter, glint, atmospheric noise, turbulence, switching, etc., can create noise of high variance --- resulting in outliers; and (3) the performance of classical tests/estimators can be unstable, i.e., asymptotic variance changes.

For example, estimators and tests based on nonparametric statistics are distribution-free, i.e., independent of nature of the underlying distribution of the data and thereby possess "intrinsic robustness" property. Furthermore, robust tests/estimators lose very little efficiency and their asymptotic variance is insensitive to changes of the underlying distribution. Huber [1, 2] formally defined robustness as, what I term as gestalt robustness as "robustness implies insensitivity against small deviations from assumptions".

In order to gain a better understanding of the meaning of robustness, as applied to robust algorithms, I would like to differentiate between two classes of algorithms, viz., Type I: intrinsically robust, and Type II: extrinsically robust. Type I is an underlying property, that is, built into the construction of the algorithm; while Type II robustness is an acquired property, that is, the algorithm needs to be robustized.

Methods needed to achieve Type II algorithmic robustness are addressed in the accompanying self-contained viewgraphs, specifically highlighting robust statistical methods from a theoretical perspective, rather than using statistical methods from a physical modeling point of view. For example, applications of Type II algorithmic robustness, from both a theoretical and practical point of view, include: robust regression; minimum variance least squares; robustized stochastic approximation (e.g., parameter, quantile, density estimation, etc., applications); image feature extraction and stable image restoration; nonlinear comparator and correlator systems; and robustizing the linearized Kalman Filter to measurement noise outliers, which is detailed in the presentation. That is, the reader is referred to the viewgraphs, which provide the foundation needed to understand the expected properties, desired qualities and limitations of robust methods, statistical models, robustizing approaches, robust estimates of location, culminating in descriptions of methods used to robustize the linearized Kalman Filter to measurement noise outliers. It is at this point several questions are posed. The questions are addressed herein by providing a synopsis of Bayesian filtering/tracking algorithms to highlight the meaning of Type I methods, i.e., using intrinsic algorithmic robustness.

\section{Synopsis of Bayesian estimation: nonlinear and non-Gaussian Bayesian tracking}

It is well known, that in the Bayesian approach to recursive state estimation, the evolution of the usual state and measurement models are assumed available in probabilistic form [3-6]. Then one attempts to construct the posterior probability density function (pdf) of the evolution of the state based on all available information, including the sequence of received measurements [4]. Since the pdf embodies all available statistical information, it may be used as the complete solution to the estimation problem. As usual, the filter consists of two stages, prediction and update. The prediction stage uses the system model to predict the state pdf forward from one measurement to the next.

*ikadar@SystemsSciences.com 
The update step uses the latest measurement to modify the prediction pdf. This step is accomplished using Bayes theorem, which updates the knowledge about the state using data from the most recent measurement. Specifically, this approach uses two recurrence relations, one defined by the Chapman-Kolmogorov equation, and the other by Bayes formula, but neither can be determined analytically in general [4]. Note that there were no assumptions made on properties of the state or the measurements (i.e., linearity, and properties of the plant and measurement noise sequences).

Under certain conditions, analytic solutions exist for the KF and grid-based filters [4]. When analytic solutions are intractable, extended KF, approximate grid-based filters and particle filters (PF) can be applied to approximate the optimal nonlinear Bayes solution. Particle filters utilize Monte Carlo methods to estimate the pdf needed using forms of importance sampling [4-7] as well as stochastic approximation (SA) parameter and density estimation methods [8, 9]. That is, the use of SA methods, and potentially robustized SA [refer to the viewgraphs] for parameter and density estimation within the PF framework, is an example of coupling Type I and Type II algorithmic approaches.

Its interesting to note here, in reference to an example given in the associated viewgraphs, that a Bayesian PF based tracking method was compared with an Interacting Multiple Model (IMM) tracker, and applied to estimating target state in the presence of heavy-tailed time-correlated non-Gaussian glint noise in 1999 [10]. A similar problem was also addressed using a Type II (extrinsically robust) robustized linearized KF tracker using Huber's M-estimator (as noted in reference [13] listed in the associated viewgraphs) by Hewer, Martin and Zeh, "Robust Preprocessing for Kalman Filtering of Glint Noise”, in 1987. The author did not compare the efficacy of the two approaches. The point here is that in some applications there is room for both Type II and Type I approaches, as discussed above, even though the Type I method is currently in the mainstream because of its generality. However, as pointed out in [5] PF methods are not to be used indiscriminately (even in some nonlinear problems), and one needs to take into account computational complexity of particle filters and the nature of the problem.

\section{CONCLUSIONS}

As exemplified above, extensions of robustized linearized Kalman Filtering methods based on Huber's M-estimator, and on the nonparametric B-L-N approach, depicted in the associated viewgraphs, could potentially be used in selected practical tracking applications when the measurement sequence is either a-priori unknown or deviates from the usual Gaussian assumption. These tracking filters can be realized with low computational complexity. Nonlinear/NonGaussian Bayesian tracking filters [3-6], and IMM tracking filters (if models are available a-priori) are clearly applicable to all cases, but can be computationally complex and not always necessary [5].

In summary the meaning of robustness is highly subjective and problem/applications dependent. It is conjectured that achieving it requires a combination of Type I and Type II algorithmic approaches. Clearly further research is needed in this complex and sometimes misunderstood area.

\section{REFERENCES}

[1] P. J. Huber, Robust Statistics, John Wiley, New York, 1973, 1981 and 2004.

[2] P. J. Huber, Robust Statistical Procedures, SIAM Series 27, 1977, $2^{\text {nd }}$ Ed. 1996.

[3] L. D. Stone, C. A. Barlow and T. L. Corwin, Bayesian Multiple Target Tracking, Artech House 1999.

[4] M. S. Arulampalam, S. Maskell, N. Gordon and T. Clapp, “A Tutorial on Particle Filters for Online Nonlinear/Non-Gaussian Bayesian Tracking” IEEE Trans. Signal Proc., Vol. 50, February 2002.

[5] B. Ristic, S. Arulampalant and N. Gordon, Beyond the Kalman Filter: Particle Filtering for Tracking Applications, Artech House 2004.

[6] A. Doucet and A. M. Johansen, “A Tutorial on Particle Filtering and Smoothing Fifteen Years Later” Tech. Report, Dept. of Statistics, University of British Columbia, December 2008.

[7] A. Doucet, N. Defreitas and N. Gordon, Sequential Monte Carlo Methods in Practice, Springer-Verlag 2001.

[8] A. Doucet and V. B. Tadic, "On-line Optimization of Sequential Monte Carlo Methods using Stochastic Approximation”, Proc. American Control Conference, May 2002.

[9] B. L. Chan, A. Doucet and V. B. Tadic, “Optimum Particle Filters Using Simultaneous Perturbation Stochastic Approximation”, Proc. International Conference on Information Fusion, 2003.

[10] N. Gordon and A. Whitby, "Bayesian Approach to Guidance in the Presence of Glint”, Journal of Guidance and Control and Dynamics, Vol. 22, May-June 1999. 


\title{
On Robust Estimation: Methods, Applications and Challenges
}

\author{
Ivan Kadar \\ Interlink Systems Sciences, Inc. \\ Lake Success, NY
}

13 April 2009

Invited Panel Discussion

Issues and Challenges in: (1) Robust Methods in Tracking, Fusion, and Decision

Making, and (2) Sensor Bias Estimation and Data Fusion with Applications to Real-World Problems

SPIE Conference 7336

"Signal Processing, Sensor Fusion and Target Recognition XVIII"

Orlando, FL April 13-15, 2009

\section{Robust Estimation - Outline}

-Why study robustness and what is meant by it?

- Potential applications (with subsequent examples on robustizing a Kalman Filter to measurement noise outliers)

- Expected properties and desired qualities of robust methods

- Statistical models

- Robustizing approaches

- Robust estimates of location

- Methods of robustizing a Kalman Filter

- Issues and Challenges 


\section{Robust Estimation}

- Motivation: Why Study Robustness?

- One never has very accurate knowledge of underlying CDF's

- Natural phenomena and man-made disturbances are rarely Gaussian, e. g., clutter, atmospheric noise, turbulence, switching, etc., can create noise of high variance --- resulting in outliers

- Performance of classical tests/estimators can be unstable, i.e., asymptotic variance changes

- Robust tests/estimators lose very little efficiency and their asymptotic variance is insensitive to changes of the underlying CDF

- "Gestalt" Robustness implies insensitivity against small deviations from assumptions (Huber)

\section{Potential Applications and Expected Properties of Robust Methods}

- Candidate Applications - Theory \& Practice

- Robust regression

- Minimum variance least squares

- Robustized stochastic approximation: e.g., parameter, quantile, density estimation,....

- Image feature extraction and stable image restoration

- Nonlinear comparator and correlator systems

$\Rightarrow$ - Robustizing the Kalman Filter to measurement noise outliers?

$-\ldots$

- Expected Properties of Robust Methods

- Efficient

- Real-time

- Computationally efficient (i. e., recursive)

- Easy to implement

- Theoretically justifiable with minimum assumptions -axiomatic

xxviii 


\section{Desired Qualities of Robust Estimators}

- Consistent $\rightarrow$ converge in probability

- Unbiased

- Equivariant in location and scale $\rightarrow$ estimates unchanged under transformations of measurements and parameters

- Asymptotically Normal

- Efficient

- Absolute: Fisher Information/Cramer-Rao Bound sense

- Relative: ratio of asymptotic variances

- Measures of Rate of Convergence - Distance Measures

- ARE: relative efficiency of two estimators/test in the limit as the samplesizes approach infinity given fixed performance parameters

- RSNR: Relative sample number ratio to reach equal asymptotic variances

- Min-Max Robust: minimize maximum asymptotic variance

- Minimum sample asymptotic "prewhitening" property

\section{Descriptive Models}

- Parametric Models - Natural Parameters and Measures:

- Expectation, Variance, Higher order Moments, Location, Scale, Skewness, Kurtosis

- Nonparametric Neighborhood models

- Parametric model with small nonparametric admixture, e. g., epsilon contaminated class, $\vartheta_{\varepsilon}$ (or "Huber-Tukey Distortion" - as defined by Kharin, i.e., includes Huber's theory of inference, Tukey's winsorizing, etc.),

$\vartheta_{\varepsilon}=[F / F=(1-\varepsilon) \phi+\varepsilon H, \varepsilon$ fixed and $0 \leq \varepsilon \leq 1, \mathrm{H}$ symmetric $]$

- Nonparametric Models with Natural Parameters

- Smoothness and symmetry conditions on unknown univariate class of CDFs

- Neighborhoods of nonparametric models with natural parameters

- Unknown symmetric CDFs with a slight amount of asymmetric contamination 


\section{Examples of Robustizing Approaches}

\section{- Bayesian Methods}

-Noise statistics are known, but non-Gaussian, estimators can be developed in a Bayesian framework, wherein observations are used to update modeled prior information. Parameters can be chosen with respect to a performance criterion, e.g., risk.

- Nonparametric Methods

- Noise statistical properties are either unknown or only partially known, distribution-free estimators can be used.

\section{- Min-Max Methods}

- Noise statistics are either incompletely known or unknown, "nature" to chose a class of cdfs, and derive an estimator whose worst case performance is optimal. If a saddle point property exists, these estimators are min-max robust.

- Combination of Min-Max and Nonparametric Methods

- Guarantees predictable (i.e., known) asymptotic properties of the resultant estimators

\section{Min-Max Robust Estimates}

- For a class of estimates, $\tau$, and the class of CDFs, $\Xi$, let $V(T, F)$ be the asymptotic variance of the estimate, when $T \in \tau$ and $F \in \Xi$. Every $F \in \Xi$ has an absolutely continuous pdf, $f$, such that the Fisher Information, $I(F)$ is bounded.

- If $\exists \mathrm{F}_{\mathrm{o}} \in \mathrm{F}$ such that $\mathrm{I}\left(\mathrm{F}_{\mathrm{o}}\right) \leq \mathrm{I}(\mathrm{F}) \forall \mathrm{F} \in \Xi$ and if $\Psi_{0} \equiv-\mathrm{f}_{\mathrm{o}} / \mathrm{f}_{\mathrm{o}} \leftrightarrow \mathrm{T}_{\mathrm{o}} \in \tau$, then $\exists \mathrm{a}$ saddle point pair $v\left(T_{0}, F_{0}\right)$ (i.e., we choose $T \in \tau$ and nature chooses $F \in \Xi$ ),

$$
\sup _{F \in \Xi} v\left(T_{0}, F\right)=v\left(T_{0}, F_{0}\right)=I^{-1}\left(F_{0}\right)=\inf _{T \in \tau} v\left(T, F_{0}\right)
$$

where, $F_{0}$ is called the least favorable CDF, and $T_{0}$ a min-max robust estimate, and $\Psi_{0}$ the most robust nonlinearity, "i.e., the worst case performance of the estimator is optimal under all $\mathrm{F} \in \Xi$, as the outcome of a game with $v(T, F)$ as the payoff"

- Important consequences for the epsilon contaminated class, $\vartheta_{\varepsilon}$ :

- M-estimates and R-estimates satisfy min-max (minimax) property

- Stochastic Approximation (SA) estimates satisfy minimax property

- Robustized SA estimates are asymptotically equivalent to $M$ or $R$ estimates 


\section{Robust Estimates of Location - Huber's M-estimator}

- Given $x_{1}, \ldots x_{n}$, i..i.d.r.v with known normalized standard deviation, CDF not exactly known, but symmetric, a sequence of M-estimators are solutions of $\alpha_{n}$

$$
\sum_{i=1}^{n} \Psi\left(x_{i}-\alpha\right)=0, \alpha=\alpha_{n}\left(x_{1}, \ldots, x_{n}\right), n=1,2, \ldots
$$

- where $\Psi(-x)=\Psi(x)$ is an odd symmetric and monotonic nonlinearity, the estimate is asymptotically normal, with zero mean and variance $\mathrm{v}_{\mathrm{M}}(\mathrm{T}, \mathrm{F})$ Depending on the chose of $\Psi$,e.g., $\Psi(x, \alpha)=(\partial / \partial \alpha) \rho(x ; \alpha)$, $\rho(x ; \alpha)=-\log f(x ; \alpha)$ one gets the ML estimate, where, $f(x ; \alpha)$ is the pdf

- $\alpha_{n}$ are also solutions of Robustized Stochastic Approximation, viz.,

$$
\alpha_{n+1}=\alpha_{n}-\frac{A_{n}(.)}{n} \Psi\left(x_{n}-\alpha_{n}\right)
$$

- M-estimators satisfy the minimax property, for the class $\vartheta_{\varepsilon}$ with $\Psi_{0}=-f^{\prime} / f_{0} \subset \Psi$

$$
\mathrm{v}_{\mathrm{M}}\left(\Psi, \mathrm{F}_{\mathrm{o}}\right) \leq \mathrm{v}_{\mathrm{M}}\left(\Psi_{\mathrm{o}}, \mathrm{F}_{\mathrm{o}}\right)=\mathrm{I}\left(\mathrm{F}_{\mathrm{o}}\right) \leq \mathrm{v}_{\mathrm{M}}\left(\Psi_{\mathrm{o}}, \mathrm{F}\right)
$$

where, $f_{o}(p d f)$ is Gaussian in the middle and double exponential at the tails.

- $\Psi_{\mathrm{o}}(\mathrm{x})$ can be called a "light limiter influence function (LLIF)"

- Issues - how to select break points?

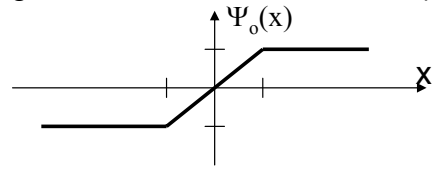

\section{Robust Estimates of Location - R-estimators}

- R-estimators are based on rank tests, and can be formulated in a general form in terms of linear rank tests, such as the two-sample Mann-WhitneyWilcoxon Nonparametric (MWWNS) test statistics, W.

- Given $x_{1}, \ldots, x_{m}$ and $y_{1}, \ldots, y_{n}$ ordered i.i.d samples from continuous and not necessarily symmetric CDFs, $F(x)$ and $G(x)=F(x-\alpha), W$, to test the hypothesis of $\alpha=0$ vs. $\alpha \neq 0$, is given by:

$$
W=\frac{1}{m n} \sum_{i=1}^{m} \sum_{j=1}^{n} \operatorname{sgn}\left(x_{i}-y_{j}\right), N=m+n
$$

- Properties under $\mathrm{H}:[\mathrm{F}(\mathrm{x})=\mathrm{G}(\mathrm{x})]$ :

- $E(W)=0$

- $V_{W}=(n+m+1) /(3 m n)$, which is independent of the underlying CDF

$$
\lim _{N \rightarrow \infty} P\left[\frac{W-E(W)}{\sqrt{V_{W}}} \leq t\right]=\Phi(t)
$$

with asymptotic Normality reached with $m=n \cong 8$ batch samples.

- Therefore, $\mathrm{W}$ is minimum sample asymptotic "prewhitening" robust unlike the M-estimator

-W also satisfies equivariance property - scale invariant unlike M-estimator

-W, as robustizing nonlinearity, satisfies minimax property for the $\vartheta_{\varepsilon}$ class, 


\section{Robustizing a Kalman Filter: Motivation}

-Why "robustize" a discrete-time Kalman Filter (KF) to outliers?

- KF assumes measurement and plant noise sequences are AWGN

- The innovations are assumed nominally Gaussian

- The distribution of the innovations can become non-Gaussian if,

- either the measurement or plant noise is non-Gaussian

- or the target dynamics are not properly modeled.

- What is done about it? (e.g.):

- Chi-squared test of the innovations (identify non-Normality) and Gate

- Estimate density of innovations and gate as needed

- Estimate quantiles of the innovations and gate as needed

- Censor (screen) measurement outliers (does not test for non-Normality, but reduces outlier magnitudes) and/or preprocess measurements prior to updating

- Interacting Multiple Models (IMM) and Particle Filters - with a-priori knowledge of noise characteristics

$-\ldots$

- Therefore, if possible?, "robustize" directly the KF against measurement noise outliers for the epsilon contaminated class

\section{Robustizing a Kalman Filter: Methods}

Consider the standard KF measurement and state linear models, under the usual AWGN assumption

$$
\begin{aligned}
& y_{k}=H_{k} x_{k}+v_{k}, E\left(v_{k} v_{j}^{T}\right)=R_{k} \delta_{k j} \\
& x_{k}=\phi_{k, k-1} x_{k-1}+w_{k-1}, E\left(w_{k} w_{j}^{T}\right)=Q_{k} \delta_{k j}
\end{aligned}
$$

The estimate $\hat{x}_{k}$ is formed at stage $\mathrm{n}$ by prediction, $\bar{x}_{k}=\phi_{k, k-1} \widehat{x}_{k-1}$ corrected by a term, viz., $\hat{x}_{k}=\bar{x}_{k}+K_{k}\left(y_{k}-H_{k} \bar{x}_{k}\right)$

$K_{k}$ is selected to minimize $P_{k}=E\left[\left(\hat{x}_{k}-x_{k}\right)\left(\hat{x}_{k}-x_{k}\right)^{T}\right], K_{k}=P_{k}{ }^{\prime} H_{k}{ }^{T}\left(H_{k} P_{k}{ }^{\prime} H_{k}{ }^{T}+R_{k}\right)^{-1}$

$\Rightarrow$ Suppose $v_{k}$ is heavy tailed (outliers) and $w_{k}$ is an AWGN sequence, then

by both intuitively and based on Huber's M-estimate of location, one would want to attenuate outliers, i.e., $\Psi\left[y_{k}-H_{k} \bar{x}_{k}\right]=\Psi\left[v_{k}\right]$ where, $\Psi$ is the LLIF applied component-wise. Given certain symmetry and continuity requirements on $F_{v} \in \vartheta_{\varepsilon}$, the estimator, $\hat{x}=\bar{x}+P_{o}^{\prime} H^{T} T^{T} \Psi\left(v^{*}\right)$ yields a bounded $P$, $P=E\left[(\hat{x}-x)(\hat{x}-x)^{T}\right] \leq P * \quad$ where, $\Psi\left(v^{*}\right)=\Psi[(y-T H \bar{x})]$, provided a transformation, $\mathrm{T}$, (to scale and symmetrize the pdf of the innovations) can be found to guarantee given conditions on $F_{v} \in \vartheta_{\varepsilon}$. The above method of Masreliez and Martin, will be termed the Linear-Nonlinear (L-N) approach. 


\section{Robustizing a Kalman Filter: Issues \& Methods}

- Issues with the L-N approach

- $T$ cannot be found directly - requires a parallel estimate, if $T$ exists

- Heuristic LLIF break points selection

- In tests yielded biased results

- An alternate method: The Linear-Batch-Nonlinear (L-B-N) approach

- Uses equivalence relationships between least squares regression and the Kalman filter

- Uses equivalence relationship between robustized stochastic approximation and linear regression

- Uses a combination of Min-Max and Nonparametric Methods under the epsilon contaminated class

- Uses the two-sample Mann-Whitney-Wilcoxon Nonparametric Statistics (MWWNS), W, applied component-wise to the innovations

- Avoids ambiguity in selecting $T$

- Guarantees small sample asymptotic Normality

- Insensitive to changes of the underlying CDF

- What does it costs? 8-10 batch sample storage and delay

\section{The L-B-N Robustized Discrete-time Kalman Filter}

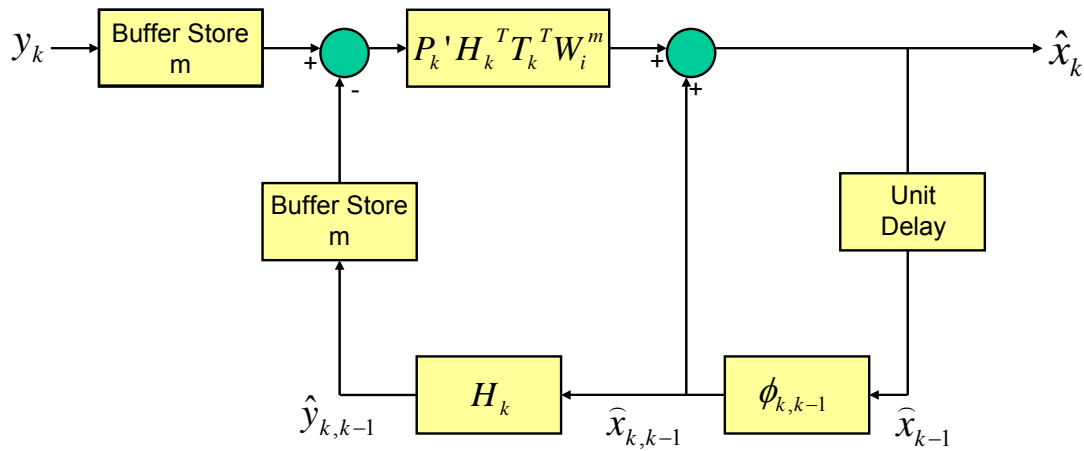




\section{Comparison of State Estimates Gaussian Mixture Noise}

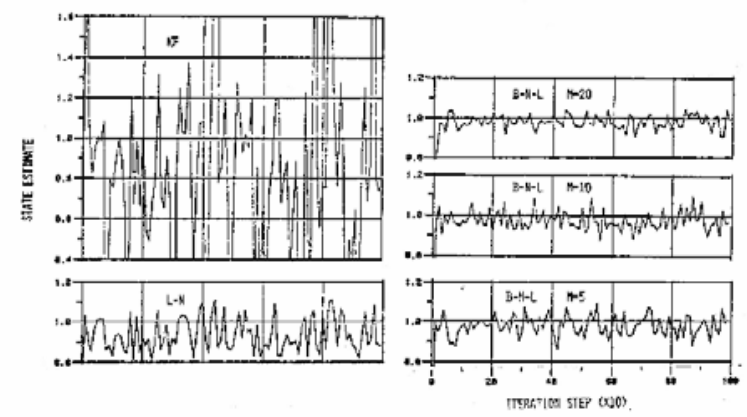

\section{Robustizing a Kalman Filter: Background}

- History of evolution of potential methods:

- Y. C. Ho of Harvard, classic report (1962) and paper (1963):

"On the Stochastic Approximation Method and Optimal Filtering Theory", J. Math. Annal. Appl. Vol. 6, pp. 152-154.

- The above work served as basis to establish equivalence between stochastic approximation, linear least squares regression and KF.

- The Matrix Inversion Lemma,

$$
(A+U C V)^{-1}=A^{-1}-A^{-1} U\left(C^{-1}+V A^{-1} U\right)^{-1} V A^{-1}
$$

establishes the equivalence between the KF and the solution of a weighted least squares model thereof.

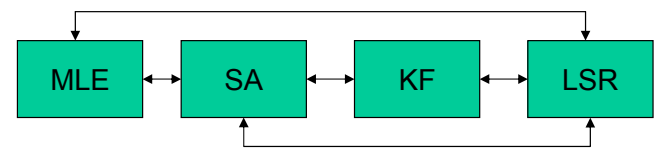

- Combining the foregoing with Huber's seminal work ushered in several potential approaches towards robustizing the KF.

xxxiv 


\section{Robustizing a Kalman Filter: Issues, Methods and Challenges}

- Issues with the L-B-N approach

- The output of the $W$ statistics is independent of the underlying CDFs, i. e., $E(W)=0$ and $\operatorname{Var}(W)=v_{w}=(2 m+1) / 3 m^{2}$, where $m$ is the batch size

- The distribution-free aspect of $W$ implies that the statistics of the robustized innovations are implicitly dependent on the measurement noise statistics, with outliers eliminated

- Additional Methods:

- Boncelet and Dickinson (1983) proposed a method talking advantage of the equivalence between the KF and a particular least-squares regression, and applied Huber's M-estimator of location to the innovations,

- Durovic and Kovacevic (1999), not aware of the above work, essentially used the same approach, except formed an estimator of the scale of the data (unlike Masreilez and Martin) using Huber's M-estimator of location,

- Kirlin and Moghaddamjoo (1986) use a median-based method to preprocess measurements prior to updating the KF with applications to target tracking

- Hewer, Martin and Zeh (1987) used Huber's M-estimator for an angle tracking Kalman Filter in glint noise; ---etc., etc., other potential papers?

- Does a practical general robustizing method exists for the linearized KF ? Bayesian estimation methods have been shown to be intrinsically "robust" to changes in the underlying assumptions (i.e., in the face of unknown i.i.d. measurement and process noise, and nonlinearities).

\section{Representative References}

[1] Y. Kharin, Robustness in Statistical Pattern Recognition, Kluwer Academic Publishers, 1996. [2a] P. J. Huber, "Robust Estimation of a Location Parameter", Ann. Math Stat. Vol. 35, 1964.

[2a] P. J. Huber, Robust Statistics, John Wiley, New York, 1973, 1981 and 2004.

[2b] P. J. Huber, Robust Statistical Procedures, SIAM Series 27, 1977; 2 ${ }^{\text {nd }}$ Ed. 1996.

[3] H. Robbins and S. Monroe, "The Stochastic Approximation Method", Ann. Math. Stat. V22, 1951

[4] Y. C. Ho, "On the Stochastic Approximation Method and Optimal Filtering Theory", J. Math. Annal. Appl. Vol. 6, pp. 152-154, 1963.

[5] M.T. Wasan, Stochastic Approximation, Cambridge University Press, 1969 and 2004.

[6] J. Tukey, Exploratory Data Analysis, Addison Wesley, Reading, MA 1977.

[7] J. Dickinson Gibbons, Nonparametric Statistical Inference, McGraw Hill Book Company, 1971.

[8] C. J. Masreliez and R. D. Martin, "Robust Bayesian Estimation for the Linear Model and Robustifying the Kalman Filter", IEEE Trans. Automatic Control, Vol. AC-22, No. 3, June 1977 [9] I. Kadar, "Robust Recursive Algorithms in Estimation and Image Processing," IBM Research Report, T. J. Watson Research Center, Yorktown Heights, NY, March 1981.

[10] C. G. Boncelet and B. W. Dickinson, "An Approach to Robust Kalman Filtering", 1983 IEEE Conference on Decision and Control, pp.304-305

[11] Z. M. Durovic and B. D. Kovacevic, "Robust Estimation with Unknown Noise Statistics", IEEE Trans. Automatic Control, Vol. AC-44, No. 6, June 1999.

[12] R.L. Kirlin and A.Moghaddamjoo, "Robust Adaptive Kalman Filtering for Systems with Unknown Step Inputs and Non-Gaussian Errors", IEEE Trans. ASSP, Vol. 34, April 1986.

[13] G. A. Hewer, R.D. Martin and J, Zeh, "Robust Preprocessing for Kalman Filtering of Glint Noise", IEEE Trans. Aerospace and Electronic Systems, AES Vol., 23, January 1987.

[14] R. Maronna, R. D. Martin and V. Yohai, Robust Statistics: Theory and Methods, John Wiley, New York, 2006.

[15] H. L. Van Trees, Detection, Estimation and Modulation Theory, Part I, John Wiley, New York 1968.

[16] Y. Bar-Shalom, X. R. Li and T. Kirubarajan, Estimation with Applications to Tracking and Navigation: Algorithms and Software for Information Extraction, J. Wiley and Sons, 2001 


\title{
Robustness, Unknowability, and Joe Btfsplk
}

\author{
Position Paper: Panel on Issues and Challenges in: \\ (1) Robust Methods in Tracking, Fusion, and Decision Making, and \\ (2) Sensor Bias Estimation and Data Fusion, with Applications to Real-World Problems \\ Ronald Maher \\ Lockheed Martin MS2 Tactical Systems, Eagan MN
}

March 23, 2009

Robustness is a central issue in the development of information fusion algorithms. In this paper I argue that when scenarios involve uncertainty due to ignorance, the best that one can do is to construct probabilistically defensible strategies for hedging against against that uncertainty. More specifically, I argue that modeling tools such as fuzzy logic and Demspter-Shafer theory may be necessary in such situations, but within the unified and Bayesian framework described in Chapters 3-8 of Statistical Multisource-Multitarget Information Fusion. ${ }^{9}$

First, what do we mean when we say that some algorithm is "robust"? An algorithm is robust, according to the most common intuitive usage, if its performance "degrades gracefully" as the uncertainty in a scenario increases. One could ask, for example, how rapidly the localization error $d(\sigma)$ of an extended Kalman filter will increase as sensor noise $\sigma$ is increased (with all other factors held constant). Filter performance degrades linearly if $d(\sigma) \cong K \sigma$ for some range of values of $\sigma$, for some constant $K$. It degrades quadratically if $d(\sigma) \cong K \sigma^{2}$. In general, if $\rho$ with $d(\sigma) \cong K \sigma^{\rho}$, then $\rho$ provides a reasonable measure of robustness.

This usage does not exhaust the common meanings of "robustness." In particular, it implicitly presumes that the phenomenon causing uncertainty is statistical and therefore that the magnitude of uncertainty can be easily quantified. Unfortunately, this is not always the case. Most forms of uncertainty are due not to randomness, but to some combination of randomness and ignorance. The following examples illustrate forms of uncertainty in which ignorance plays an increasingly greater role:

1. Randomness with slight confusion: Noise that consists of a dominant random process, contaminated by a sporadic (and therefore poorly understood) secondary random process.

2. Randomness with moderate confusion: A SAR image that is difficult to interpret because of statistically uncharacterizable variations in the observed targets due to mud, turret articulation, nonstandard placement of equipment on the target surface, etc.

3. Randomness with considerable confusion: Two sensors whose observations are correlated because of a common but uncharacterizable underlying noise phenomenology.

4. Moderate confusion with slight randomness. A digital voltmeter returns a measurement that has the form of a nearly constant interval, say 2.0 volts to 3.0 volts.

5. Moderate confusion with some randomness: A data fusion system that, because of an uncharacterizable communications network topology, processes all measurements as though they are independent even though some or many measurements were actually originated by the same sensor or sensors. 
6. Considerable confusion with some randomness: In a great many situations it is difficult for the "sensor" to even specify what the "measurement" is, as with the following natural-language report:

$$
\begin{aligned}
\mathcal{S}= & \text { 'Gustav is probably near the tower, but it could } \\
& \text { be the smokestack, it's so foggy I can't say for sure.' }
\end{aligned}
$$

In this case the "sensor," a human observer, confronts us not only with vagueness ("near") but also with uncertainty in the form of three increasingly vague ("could be") hypotheses: (1) 'Gustav is near the tower'; (2) 'Gustav is near the smokestack'; and (3) 'I'm not sure what I'm seeing'.

The question then is this: what methods might be devised to ensure "robustness" with respect to these increasingly challenging forms of uncertainty? Some common approaches:

1. Randomness with slight confusion: apply the "epsilon-contamination" model proposed by Huber as part of his "robust statistics." 4

2. Randomness with moderate confusion: physics-based CAD modeling; class-generic probability distributions.

3. Randomness with considerable confusion: generalizations of Julier's \& Uhlmann's covariance intersection. ${ }^{5,6,8}$

4. Moderate confusion with slight randomness: techniques from statistical interval analysis, e.g., Choquet integrals. ${ }^{3}$

5. Moderate confusion with some randomness: generalizations of covariance intersection; fuzzy logic, DempsterShafer theory.

6. Considerable confusion with some randomness: fuzzy logic modeling, for example as described in Antony. ${ }^{1,2}$

As the degree of ignorance increases relative to the degree of randomness, solution methods increasingly progress from the more statistical to the more heuristic. Ordinary and robust statistics lie at one extreme, and fuzzy logic and other expert-system techniques lie at the other. Let's look at both in turn.

Robust statistics ${ }^{4}{ }^{7}$ In this case one assumes that an imperfectly characterized probability distribution $f(\mathbf{z})$ on measurements $\mathbf{z}$ belongs to some suitably well-behaved, pre-specified class $\mathfrak{F}$ of functions $F(\mathbf{z})$. This family models the likelihood functions that are plausible because of uncharacterizable statistical variations. Such an approach is useful in certain circumstances. However we believe that, in general, conventional robust statistics approaches are self-contradictory because their claimed optimality presumes accurate models of ignorance. The small handful of function families $\mathfrak{F}$ normally employed in robust statistics are chosen for their mathematical tractability -i.e., the fact that it is possible to prove optimality results if one assumes them-rather than for their fidelity to the actual structure of uncertainty in any given problem. Under conditions of uncertainty due to ignorance, an optimizable uncertainty structure $\mathfrak{F}$ may bear so little resemblance to the actual but unknowable structure of uncertainty that any claim of optimality based on it is meaningless.

Fuzzy logic. This and related approaches proceed in an entirely different manner. They take the stance that the best that one can do when the structure of uncertainty is unknowable, is to construct ways of hedging against against that uncertainty. A primary difficulty with fuzzy logic and similar expert-systems approaches is their lack of generally accepted theoretical foundations. This gap has, among other things, led to interminable "expert systems wars" and to considerable confusion on the part of practitioners about what method should be applied to what situation and why.

Over the last decade I have argued that the best approach is to place one foot firmly in each camp by rigorously incorporating many expert-system approaches within a Bayesian framework. When the ignorance involved in a 
scenario is great, there can be no "optimal" ways of modeling uncertainty and producing state estimates. On the other hand, this does not mean that such matters must be abandoned to unapologetic heuristics. Instead, I have endeavored to provide solid statistical, computational, and practical foundations for supposedly heuristic methodologies. This work has been described in practitioner-level detail in Chapters 3 through 8 of Statistical Multisource-Multitarget Information Fusion. ${ }^{9}$ It is beyond the scope of this paper to describe this work in any detail. In what follows I provide a very brief summary.

The recursive Bayes filter is the theoretical foundation for single-sensor, single-target tracking and target identification. This filter relies on two fundamental a priori models. The Markov transition density $f_{k+1 \mid k}\left(\mathbf{x} \mid \mathbf{x}^{\prime}\right)$ models our best guesses about interim target motion between consecutive measurement collections. This function provides the basic information necessary to implement the Bayes filter's time-update step. The likelihood function $f_{k+1}(\mathbf{z} \mid \mathbf{x})$ describes the probability (density) that a measurement-vector $\mathbf{z}$ will be collected from a target if its state-vector at time-step $k+1$ is $\mathbf{x}$. This function provides the basic information necessary to implement the Bayes filter's measurement-update (i.e., Bayes' rule) step.

Much of my work has been devoted to showing how this basic scheme can be extended to nontraditional information. Such information includes the natural-language statement (1) above, as well as attributes, features, and inference rules. First, I have shown that such information can be modeled as random subsets $\Theta$ of the underlying sensor/observer measurement space. Second, I have shown how to extend $f_{k+1}(\mathbf{z} \mid \mathbf{x})$ to "generalized likelihood functions" of the form $f_{k+1}(\Theta \mid \mathbf{x})$. Third, I have shown how familiar expert-systems uncertainty modeling methods (fuzzy logic, Dempster-Shafer inference, rule-based inference) can be applied to nontraditional information and then put into random set form $\Theta$. Given this, nontraditional information of all kinds can be processed in a manner that is fundamentally no different than that used to process, say, radar detections. This formal process provides, for example, a rigorous and systematic means of implementing the more heuristiclydescribe process devised by Antony. ${ }^{1,2}$ Finally, I show how this single-source, single-target methodology can be rigorously extended to multisource-multitarget scenarios via the multitarget recursive Bayes filter and its approximations, the PHD and CPHD filters.

The end result is a systematic Bayesian approach to robustness, especially in regard to nontraditional measurements and their processing. On the one hand, measurement fusion using fuzzy conjunction, Dempster's rule of combination, and rule-based inference can be shown to be equivalent to Bayes' rule. ${ }^{9, p p .181-189}$ As just one example, fuzzy conjunction of fuzzy measurements $g, g^{\prime}$ can be shown to obey the following relationship: $f_{k \mid k}\left(\mathbf{x} \mid g \wedge g^{\prime}\right)=f_{k \mid k}\left(\mathbf{x} \mid g, g^{\prime}\right)$. (Here, $f_{k \mid k}(\mathbf{x} \mid E)$ denotes a Bayes posterior distribution on target state $\mathbf{x}$, conditioned on evidence $E$ available as of time-step $k$.) On the other hand, information-update using Dempster's rule can be shown to be a special case of Bayes' rule. ${ }^{9}$,pp.199-209

Despite this fact, my approach still tends to encounter resistance, in part because of the "expert system wars" previously mentioned. There is a tendency on the part of some people, especially those with Bayesian inclinations, to stop listening the moment that words such as "fuzzy logic" or "Dempster's combination" are uttered. In response to such reactions I offer the following hypothetical argument. Suppose that, decades ago, a rather eccentric researcher by the name of Dr. Joe Btfsplk ${ }^{1 \S}$ introduced a data fusion approach that has since become known as "Btfsplk's rule of combination." Let measurements $\mathbf{z}_{1}, \mathbf{z}_{2}$ be given with associated covariance matrices $C_{1}, C_{2}$. Then Btfsplk combination is defined as

$$
\left(C_{1}, \mathbf{z}_{1}\right) \otimes\left(C_{2}, \mathbf{z}_{2}\right)=(C, \mathbf{z})
$$

where

$$
C \triangleq\left(C_{1}^{-1}+C_{2}^{-1}\right)^{-1}, \quad \mathbf{z} \triangleq\left(C_{1}^{-1}+C_{2}^{-1}\right)^{-1}\left(C_{1}^{-1} \mathbf{z}_{1}+C_{2}^{-1} \mathbf{z}_{2}\right) .
$$

Being the jinxed individual that he was, Dr. Btfsplk managed to alienate a great number of people, probabilists especially, by boasting that Btfsplk combination represented a radically new and even "non-Western" mode of

\footnotetext{
${ }^{1 \S}$ Readers of a certain age and nationality will recognize this as being the hapless, jinxed character in the now-defunct American newspaper cartoon strip "Lil' Abner." Some have claimed that the name is pronounced "bitzfelsplik."
} 
"nonlinear logic." As a consequence, many individuals simply refuse to listen the instant that the name "Btfsplk" is even mentioned.

But is this a defensible stance? As most readers will realize, "Btfsplk combination" is - whatever its original provenance - actually a form of Bayes' rule under linear-Gaussian assumptions. ${ }^{9, p .112}$ My research has shown that the same thing is true of fuzzy conjunction, Dempster's combination, and the "firing" of inference rules. Consequently, there is no justification for "Btfsplkophobia" under these circumstances.

\section{REFERENCES}

[1] Antony, R., and Karakowski, J., "Fusion of HUMINT and conventional multi-source data," McLean, VA, Proc. 2007 MSS Nat'l Symp. on Sensor and Data Fusion, MITRE Corp., McLean VA, June 11-14 (2007).

[2] Antony, R., and Karakowski, J., "Towards greater consciousness in data fusion systems," Proc. 2007 MSS Nat'l Symp. on Sensor and Data Fusion, McLean, VA, June 11-14 (2007).

[3] Grabisch, M., Nguyen, H., and Walker, E., [Fundamentals of Uncertainty Calculi With Applications to Fuzzy Inference], Kluwer Academic Publishers, Dordrecht (1995).

[4] Huber, P. J., [Robust Statistics], John Wiley \& Sons, New York (1981).

[5] Hurley, M., "An information-theoretic justification for covariance intersection and its generalizations," Proc. 5th Int'l Conf. on Information Fusion, 1, 505-511, Annapolis MD, July 7-11 (2002).

[6] Julier, S., "An empirical study into the use of Chernoff information for robust, distributed fusion of Gaussian mixture models," Proc. of 9th Int'l Conf. on Information Fusion, Florence, Italy, July 10-13 (2006).

[7] Kharin, Y., [Robustness in Statistical Pattern Recognition], Kluwer Academic Publishers, Dordrecht, (1996).

[8] Mahler, R., "Optimal/robust distributed data fusion: a unified approach," Proc. SPIE 4052, 128-138 (2000).

[9] R. Mahler, [Statistical Multisource-Multitarget Information Fusion], Artech House, Norwood, MA, 2007. 


\section{Robustness, Unknowability, and Joe Btfsplk*}

Panel on Issues and Challenges in:

(1) Robust Methods in Tracking, Fusion, and Decision Making, and

(2) Sensor Bias Estimation and Data Fusion,

with Applications to Real-World Problems

\section{Ronald Mahler}

Lockheed Martin MS2 Tactical Systems, Eagan MN

SPIE Defense, Security + Sensing Symposium

April 13 2009, Orlando

\section{The Position Advocated in this Presentation}

- When scenarios involve uncertainty due to ignorance:

- the best that one can do is to construct probabilistically defensible strategies for hedging against that uncertainty

- under such conditions, "optimal robustness" is an inherently self-contradictory term

- To address "robustness," one must first identify and differentiate between highly disparate meanings of the term

- A priori rejection of heuristic techniques such as fuzzy logic, Dempster-Shafer theory, etc., is short-sighted

- A better approach: rigorously incorporate heuristic robustness techniques into a Bayes-filter paradigm 


\section{But First, Something Completely Different}

Item: in the 1960s \& 1970s, the eccentric

Dr. Joe Btfsplk propounds a new approach

to sensor fusion: "Btfsplk combination"

Item: being a jinx to himself-as well as

everyone else-he unwisely makes

many extravagant claims:

- "a radical, non-linear form of reasoning"

-"not comprehensible to those wedded to Western forms of

logic and thought"

he thereby alienates many people-especially Bayesians

- so what is this controversial Btfsplk combination?

sensor 1 delivers measurement $\mathbf{z}_{1}$ with covariance matrix $C_{1}$

sensor 2 delivers measurement $\mathbf{z}_{2}$ with covariance matrix $C_{2}$

sensor fusion formula: $\left(C_{1}, \mathbf{z}_{1}\right) \otimes\left(C_{2}, \mathbf{z}_{2}\right)=(C, \mathbf{z})$ where $C^{-1}=C_{1}{ }^{-1}+C_{2}^{-1}$

$C^{-1} \mathbf{z}=C_{1}^{-1} \mathbf{z}_{1}+C_{2}^{-1} \mathbf{z}_{2}$

\section{Intuitive Concept of Robustness of an Algorithm}

- an algorithm is robust if it "degrades gracefully" as the uncertainty in a scenario increases

- example: how rapidly does localization error $d(v)$ of EKF increase as sensor variance $v$ is increased (all other factors held constant)?

- error "degrades linearly" if $d(v) \cong K \cdot v$ for some range of $v$

- error "degrades quadratically" if $d(v) \cong K \cdot v^{2}$ for some range of $v$

- this common usage implicitly assumes that the magnitude of uncertainty can be meaningfully quantified-e.g., $v$

- as the amount of uncertainty to to ignorance increases, the more difficult it becomes to quantify or even define robustness 


\section{Varieties of Robustness}

Randomness with slight confusion: a dominant noise process,

contaminated by a sporadic, poorly understood secondary noise process

Randomness with moderate confusion: a SAR image, difficult to interpret because of uncharacterizable target variations (mud, turret articulation, nonstandard placement of equipment, etc.)

Randomness with considerable confusion: two sensors whose

observations are correlated because of a common but uncharacterizable underlying noise phenomenology

measurement that is a nearly constant interval, say $[2.0,3.0]$

a digital voltmeter returns a

a data fusion system fed

with unpedigreed measurements (uncharacterizable double counting)

statements, e.g.:

natural-language

- "Gustav is probably near the tower, but it could be the smokestack, it's so foggy I can't say for sure."

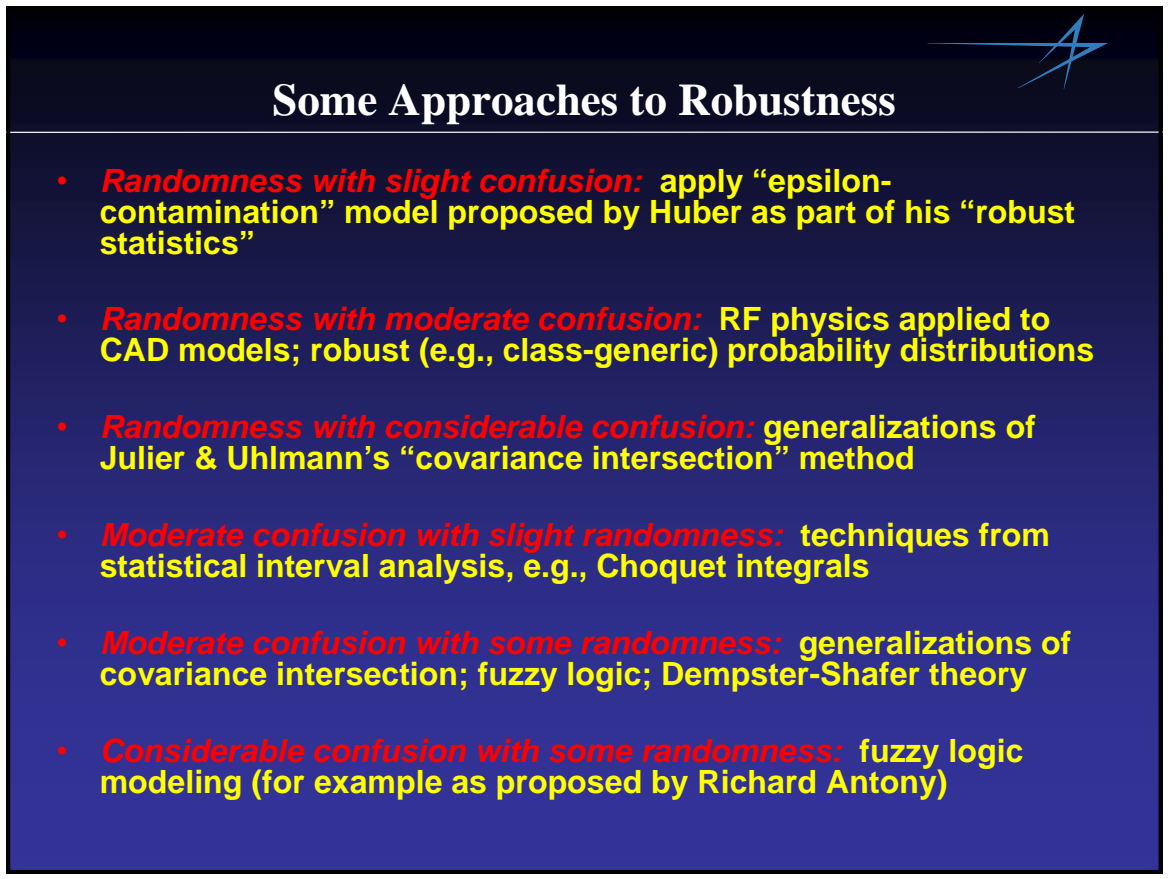




\section{“Btfsplk Combination" Revisited}

\section{Item: Btfsplk combination turns out to be a}

special case of Bayes' rule:

combination: $\left(C_{1}, \mathbf{z}_{1}\right) \otimes\left(C_{2}, \mathbf{z}_{2}\right)=(C, \mathbf{z})$

Bayes' rule: $\frac{N_{C_{1}}\left(\mathbf{x}-\mathbf{z}_{1}\right) \cdot N_{C_{2}}\left(\mathbf{x}-\mathbf{z}_{1}\right)}{\int N_{C_{1}}\left(\mathbf{w}-\mathbf{z}_{1}\right) \cdot N_{C_{2}}\left(\mathbf{w}-\mathbf{z}_{1}\right) d \mathbf{w}}=N_{C}(\mathbf{x}-\mathbf{z})$

and so it is with fuzzy logic, Dempster-Shafer theory, and rule-based inference, as methods for instilling robustness

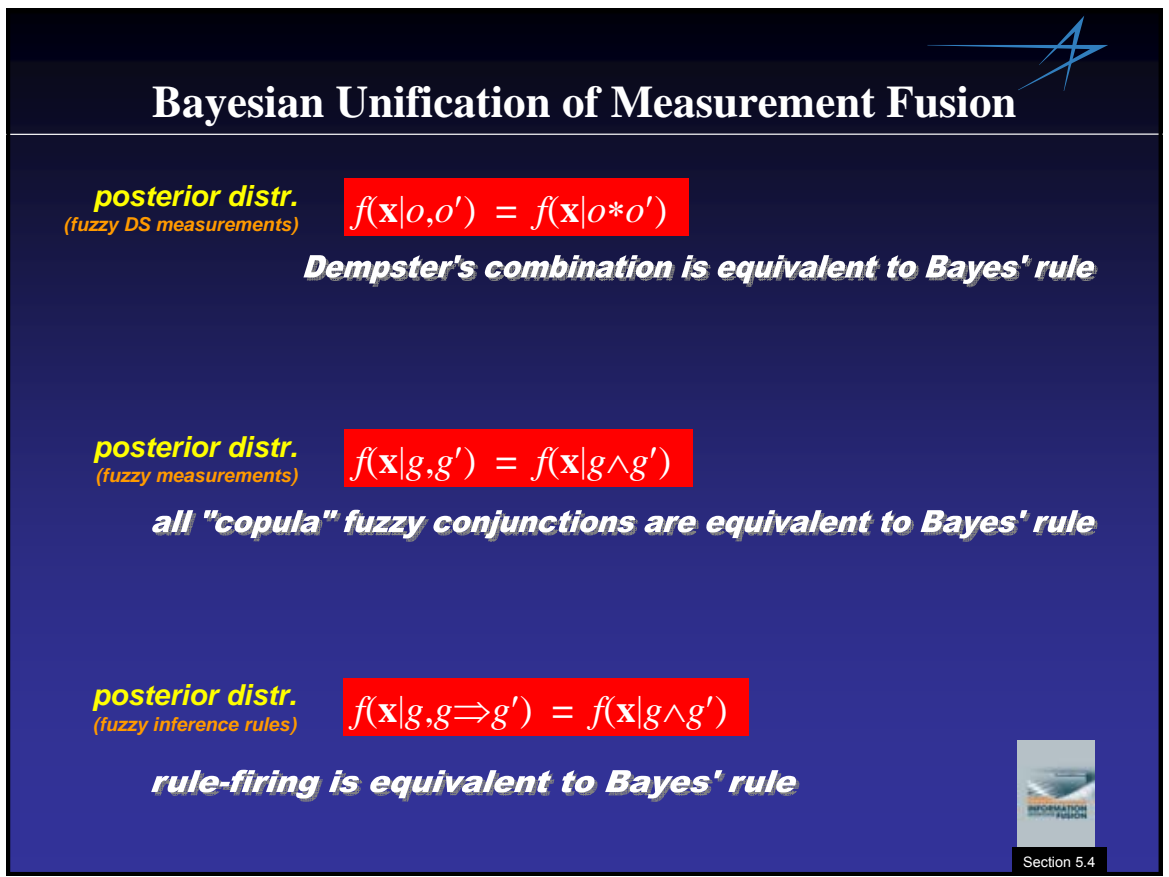




\section{Data-Update via Dempster's Rule}

Item: the data-update step of the Bayes filter reduces to Btfsplk combination if one assumes that the likelihood function has the form

$$
L_{\mathbf{z}}(\mathbf{x})=N_{R}(\mathbf{z}-H \mathbf{x}) \quad \mathbf{z}=\text { conventional measurement }
$$

and then asks, What simplified form must the Markov density and the posteriors have to produce closed-form formulas?

the data-update step of the Bayes filter reduces to combination if one assumes that the likelihood function has the form

$$
L_{o}(\mathbf{x})=\Sigma_{g} O(g) g(H \mathbf{x}) \quad o=D \text {-S measurement (e.g., natural- }
$$

and then asks, What simplified form must the Markov density and the posteriors have to produce closed-form formulas?

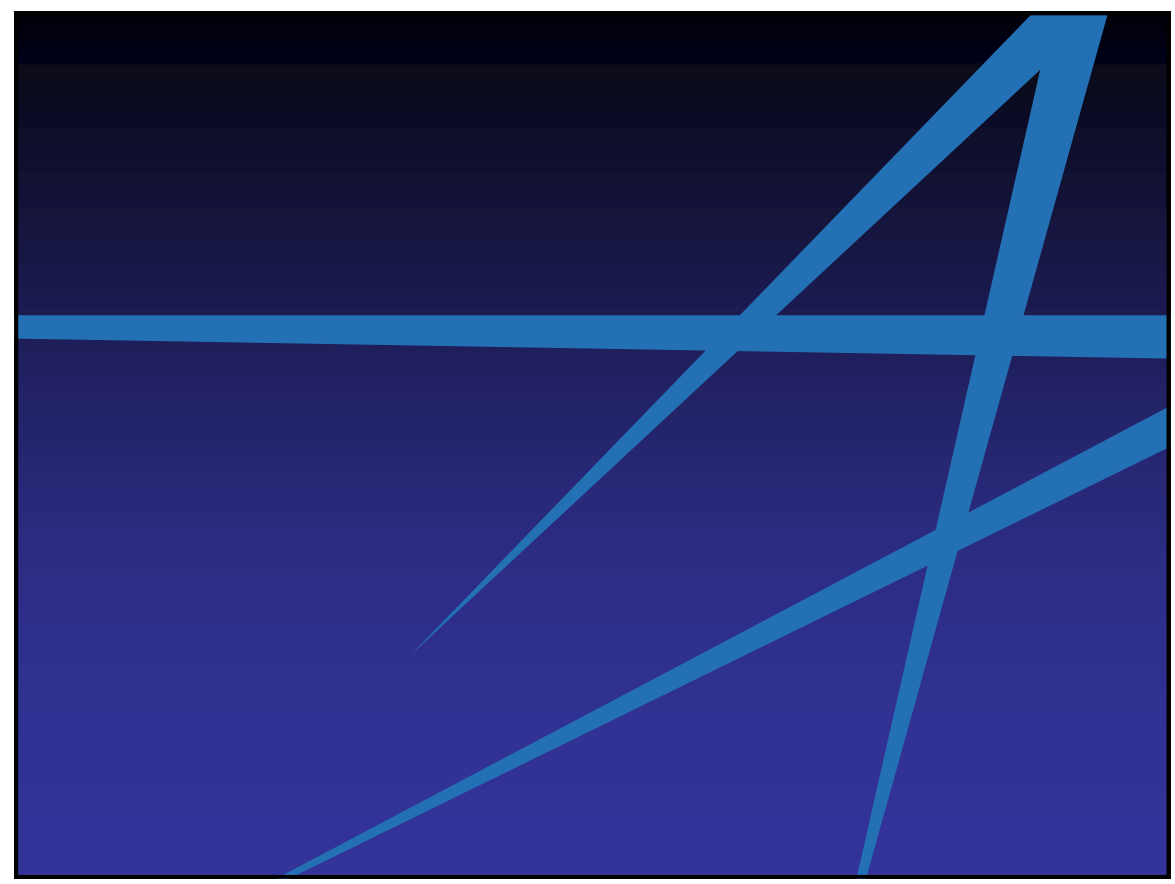




\title{
Tracking with Sensors of Opportunity: A Real-World Case Study with Bias, Clutter and Fusion
}

\author{
T. Kirubarajan \\ Estimation, Tracking and Fusion Laboratory \\ Electrical and Computer Engineering Department \\ McMaster University, Hamilton, Ontario, Canada \\ kiruba@mcmaster.ca
}

\begin{abstract}
:
In this paper, we consider Passive Coherent Location (PCL), where signals-of-convenience (e.g., radio, $\mathrm{TV}$, cellular) ared use for tracking airborne targets, as a case study to illustrate some specific real-world issues in multisensor-multitarget tracking. While most tracking algorithms assume bias-free measurements with additive white Gaussian noise and spatially uniform clutter, real data does not conform to this simplistic model. In a representative dataset considered as part of a recent study on the feasibility of PCL systems, many idiosyncrasies, including banded clutter, time-varying bias, spatially/temporally varying clutter and measurement-to-transmitter uncertainty, were observed. Here, we briefly discuss ways to address these issues in real-world problems.
\end{abstract}

\section{Introduction}

Passive coherent location (PCL) is an emerging (or a re-emerging) technology in air defence systems. PCL system uses existing commercial signals (e.g., FM broadcast and TV transmission signals) as illuminators of opportunity [2]. PCL systems have a number of advantages over conventional monostatic or bistatic radar systems. The monostatic or bistatic active radars reveal their locations. Hence, they quickly become combat targets due to their energy emission. Since there is no active radar transmission signals in PCL systems, there is almost no risk of being detected as the transmission sources are already out there in the environment for different purposes. Therefore, PCL facilitates covert operation and increases resilience to electronic countermeasures. Since FM radio and TV signals are freely available across the land, PCL systems have number of advantages such as wide coverage, low cost of operation and maintenance, and operation without a frequency clearance. The PCL system can also be used to fill dead-spots in existing radar coverage and extend the awareness of surveillance territories. Multi-illumination provides signals where mountains and valleys are present. Therefore, it becomes possible to decrease the blind zones in an economic and effective manner. TV and FM transmitters focus their broadcast energy towards the Earth's surface. These signals focus to cope with non-line-of-sight propagation and can perform better even with a very inefficient antenna and receiver systems. The FM band signals give high RCS returns. Therefore, the capability to detect low-flying stealthy targets is enhanced in PCL systems.

\section{Sample Real Dataset}

A real dataset using a bistatic PCL system (i.e., one transmitter and one receiver) was used for tracker evaluation [5]. There are multiple targets in the surveillance with most of them going along straight lines trajectories. That is, targets' motion capability is not the most challenging aspect in this data set.

\section{Identified Issues and Solutions}

One of the most striking aspects in the dataset is banded clutter. A large number of false alarms are observed in a narrow region. Like most other trackers, the Interacting Multiple Model (IMM) estimator 
combined with multiframe assignment [3] assumes spatially uniform clutter. The banded clutter results in a high number of spurious tracks and track swaps. The only computationally feasible solution for this non-uniform clutter is to estimate the clutter map online in an integrated manner with feedback to and from the tracker [1]. Other identified issues are sensor coverage and non-uniform probability of detection. While it is known that there are targets in certain areas, the receiver did not get any detection from a few targets in that region at all. Again, adaptive processing that can counter the effects of nonuniform detection are needed to address this problem.

Another significant issue was the bias in Doppler measurements. Strangely, this bias was observed for selected tracks only. That is, the bias is not just sensor dependent, but target dependent as well, which necessitates the integration of tracking and bias estimation at the track level [4]. The effect of bias was even more drastic when measurements from another sensor in the same surveillance region were used to fuse tracks from multiple receivers.

A PCL specific issue is the uncertainty in transmitter identity when multiple transmitters operating at the same frequency are in the same surveillance region (e.g., multiple radio stations or repeaters operating at the same frequency). In this case, in addition to the standard data association problem of measurement-to-target origin uncertainty, the measurement-to-transmitter uncertainty needs to be handled as well. A novel solution using multiframe assignment is presented in [6].

\section{Summary}

Real-world datasets do not confirm to the naïve assumptions of bias-free target-originated measurements with additive Gaussian noise and spatially uniform clutter. The bias and clutter models may vary over time as well as over space. In this case, adaptive techniques that can estimate clutter models, detection probabilities and biases in real-time in conjunction with the tracker are needed to handle real-world datasets.

\section{Acknowledgements:}

The assistance of Dr Tharamarasa Ratnasingam, Dr Mike McDonald and Dr Sjoerd Gelsema for this work is gratefully acknowledged.

\section{References:}

[1] X. Chen, R. Tharmarasa, M. Pelletier and T. Kirubarajan, "Integrated clutter modeling and target tracking”, Accepted for Proc. SPIE Conf. Small Target Tracking, San Diego, August 2009.

[2] P. E. Howland, D. Maksimiuk and G. Reitsma, "FM radio based bistatic radar", IEE Proceedings on Radar Sonar Navigation, Vol. 152, No. 3, June 2005.

[3] R. L. Popp, T. Kirubarajan and K.R. Pattipati, "Survey of Assignment Techniques for Multitarget Tracking”, Chapter 2 in Multitarget/Multisensor Tracking: Applications and Advances III, (Y. BarShalom and W.D. Blair, eds.), Artech House, 2000.

[4] M. Subramaniam, K. Punithakumar, M. McDonald and T. Kirubarajan, "Integrated bias removal in passive radar systems”, Proc. SPIE Conf. Signal and Data Processing of Small Targets, Vol. 6969, Orlando, March 2008.

[5] M. Subramaniam, R. Tharmarasa, M. McDonald and T. Kirubarajan, "Passive tracking with sensors of opportunity using passive coherent location”, Proc. SPIE Conf. Signal and Data Processing of Small Targets, Vol. 6969, Orlando, March 2008.

[6] R. Tharmarasa, M. McDonald and T. Kirubarajan, "Resolving transmitter origin uncertainty in PCL systems”, Accepted for Proc. SPIE Conf. Small Target Tracking, San Diego, August 2009. 
Tracking with Transmitters of Opportunity:

A Real-World Problem with Bias, Clutter and Fusion

T. Kirubarajan (Kiruba)

Estimation, Tracking and Fusion Laboratory (ETFLab)

Department of Electrical and Computer Engineering

McMaster University

April 17, 2009

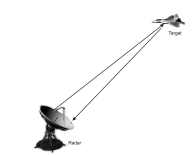

Figure: Monostatic radar

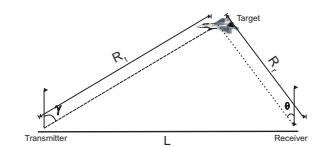

Figure: Bistatic radar

- Monostatic radar: transmitter and receiver are collocated

- Bistatic radar: transmitter and receiver are separated

- Multistatic radar: generalization of bistatic radar

- One or more receivers process returns from one or more geographically separated transmitters. 
Passive Coherent Location (PCL)

McMaster University

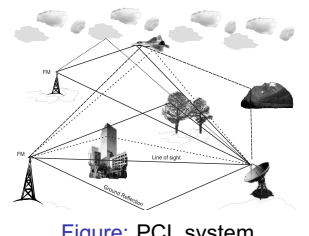

Figure: PCL system

- A specific case of bistatic/multistatic radar.

- Exploit non-radar transmitters of opportunity.

- Detect and track airborne targets by processing reflections from non-cooperative sources of illumination.

- Analog television signals

- FM radio signals

- GSM base stations

- Digital audio/video broadcasting

- High-definition television transmitters

- Satellite signals (low strength and illumination is too infrequent) 
Advantageous of PCL

- The radiation sources are already out there.

- Operate without a frequency clearance.

- Increased resilience to electronic countermeasures as waveforms and receiver locations are unknown.

- Low cost of operation/maintenance due to the lack of transmitter and moving parts.

- Target reception is at the ground level - better accuracy on low-flying targets.

- Gap filler in the radar surveillance region at low cost.

\section{Disadvantageous of PCL $\quad$ McMaster}

- No control over the transmission (e.g., frequency, average power, transmitted waveform)

- Commercial broadcasts are not designed for radar processing.

- Small antenna size (for low cost, high mobility and stealthiness) - Reduced detection range and degraded spatial resolution.

- Frequency range is affected by galactic noise.

- Other transmitters of opportunity can also contribute to higher noise levels. 


\section{Challenges}

\section{- Very low probability of detection}

- High false alarm rate

- High nonlinearity with large measurement noises and long sensor-to-target distances

- Lack of elevation information

- Hard to estimate the altitudes of targets.

- Possible measurement biases

- Need to model and estimate sensor biases.

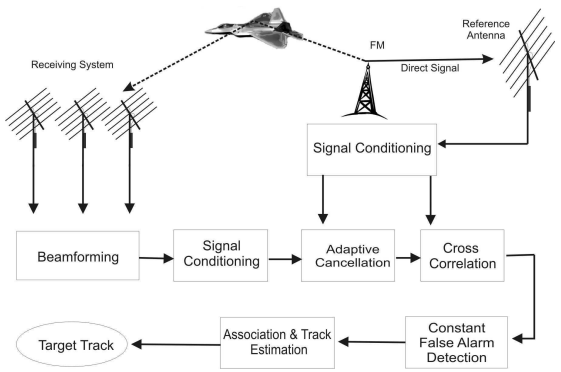

Figure: A PCL Tracking System 


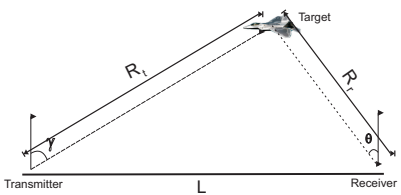

- Measurements are bistatic range, bistatic doppler shift and bearing:

$$
\left(\begin{array}{c}
r_{b} \\
f_{b} \\
\theta
\end{array}\right)=\left(\begin{array}{c}
R_{r}+R_{t}-L \\
-\frac{f}{c}\left(\dot{R}_{r}+\dot{R}_{t}\right) \\
\tan ^{-1}\left(\frac{x-x_{r}}{y-y_{r}}\right)
\end{array}\right)+b+\omega
$$

$f$ is the transmitter frequency, $c$ is the speed of wave, $b$ is the bias and $\omega$ is the noise

- Track initialization

- Track is a symbolic representation of a moving target.

- Measurement association

- 2-D assignment - Match the latest list of measurements to the

list of tracks.

- Multiframe assignment - Match the last $S-1$ lists of

measurements.

- Filtering

- Nonlinearity

- Target maneuvers

- Track maintenance

- Track confirmation

- Track deletion 

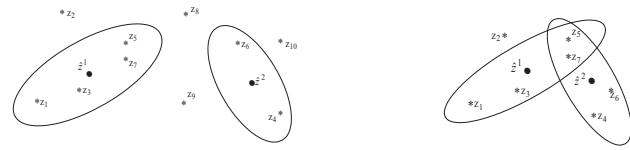

Figure: Well-separated targets

Figure: Closely spaced targets.

- Need data association to resolve measurement origin uncertainty.

- Possible approaches:

- Nearest Neighbor (NN) and Strongest Neighbor (SN)

- Probabilistic Data Association (PDA)

- Multiple Hypothesis Tracking (MHT)

- 2-D or multiframe assignment

\section{2-D Assignment}

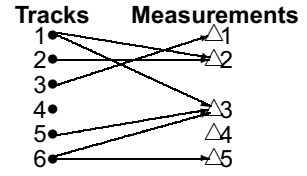

Figure: Candidate assignments

- Constraints:

- A measurement is assigned to at most one track.

- Each track is assigned to at most one measurement.

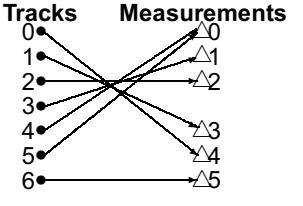

Figure: One-to-one 2-D assignments 


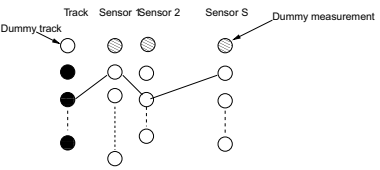

- Constraints:

Figure: Assignment tree for $(S+1)-\mathrm{D}$

$$
\begin{gathered}
\sum_{i_{1}=0}^{N_{1}} \cdots \sum_{i_{S}=0}^{N_{S}} \chi_{t i_{1} i_{2} \ldots i_{S}}=1, t=1, \ldots, T \\
\sum_{t=0}^{T} \sum_{i_{2}=0}^{N_{2}} \cdots \sum_{i_{S}=0}^{N_{S}} \chi_{t j i_{1} i_{2} \ldots i_{S}}=1, j=1, \ldots, N_{1}
\end{gathered}
$$

- Handling nonlinearity:

- Converted measurement Kalman filter (CMKF)

- Simple

- Extended Kalman filter (EKF)

- Use first order linearization around predicted target state.

- Performs poorly if linearizarion is not accurate enough.

- Unscented Kalman filter (UKF)

- No linearization

- Performs better than EKF with slightly more computation.

- Particle filter (PF)

- Best known approach with high computation.

- Difficult to handle varying number of targets.

- Probability Hypothesis Density (PHD) method

- Automatically estimates the number of targets.

- Can be implemented using particle filters 


\section{Interacting Multiple Model (IMM) Estimator}

- Handle target maneuvers

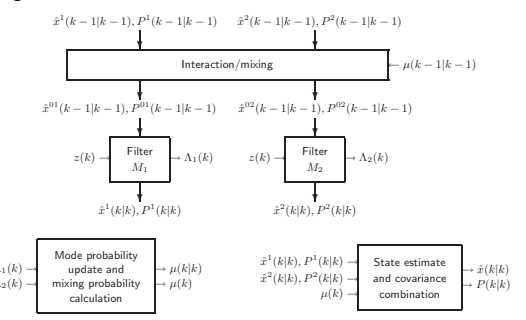

\section{Track Maintenance}

- Tracks are classified as tentative and confirmed.

- Tracks are updated using the measurements in the following order:

1) Confirmed tracks 2) Tentative tracks.

- Unassociated measurements are used to initialize new tracks

- Tracks are confirmed/deleted when confirmation/deletion rules are satisfied.

- Confirmation/deletion rules are based on either logic or quality.

- Logic: $n$ measurement out of $m$ time steps

- $n$ and $m$ are selected based on $P_{D}$ and $P_{F A}$.

- Quality: calculated based on measurement likelihood. 
- Measurement variances $\sigma_{r}^{2}=400 \mathrm{~m}^{2}$ and $\sigma_{\dot{r}}^{2}=1$

- Two targets entering at $k=1$ and $k=15$

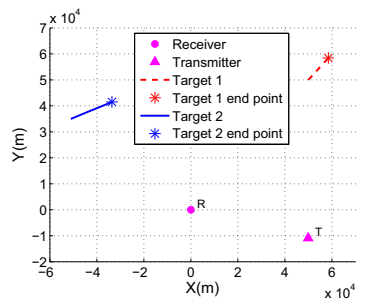

Figure: Target trajectories and locations of transmitter and receiver

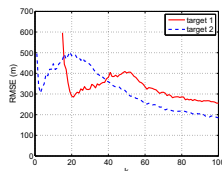

(a) CMKF

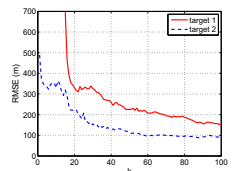

(b) UKF

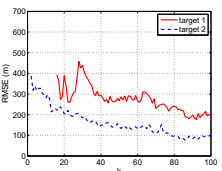

(c) PHD

Figure: RMSE comparison of filters with $\sigma_{\theta}^{2}=0.0001$ radians $^{2}$

- The performances of UKF and PHD filters are better than CMKF.

- PHD filter with 1000 particles performs similar to CMKF.

- 4000 particles are needed to match the performance of the UKF. 


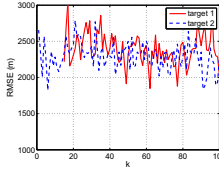

(a) CMKF

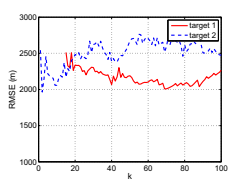

(b) UKF

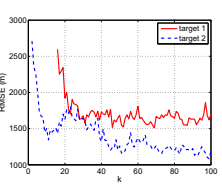

(c) PHD

Figure: RMSE comparison of filters with $\sigma_{\theta}^{2}=0.01$ radians $^{2}$

- The performance of PHD filter is better than UKF and CMKF.

- The performance of UKF was good in few runs.

- Overall RMSE is high due to track losses.

\section{Simulation Results (cont'd)}

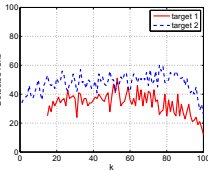

(a) CMKF

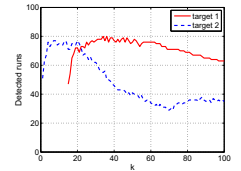

(b) UKF

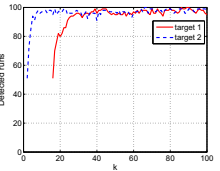

(c) PHD

Figure: Number of runs in which tracks are found within $5000 \mathrm{~m}$ distance from the target out of 100 runs with $\sigma_{\theta}^{2}=0.01$ radians $^{2}$

- PHD filter tracked the targets well in almost all the runs.

- CMKF and UKF missed the targets in several runs due to

- track breakages for CMKF.

- track losses for UKF. 


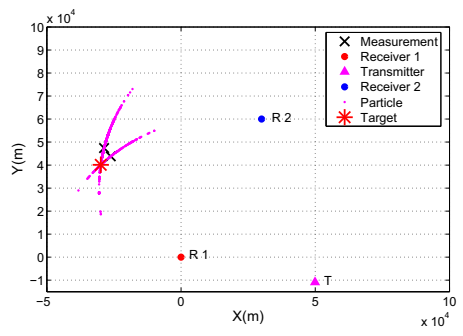

Figure: Particles at the initial time step

- Effects of bias can be mitigated by using more than one transmitter or receiver.

\section{Real Data: TNO/NATO Data (Bistatic)}

- Locations of transmitters and receivers are given in Earth Centered-Earth Fixed geodetic (ECEF-g) coordinates.

- ECEF-g coordinates are converted to local tangent plane (LTP) coordinates.

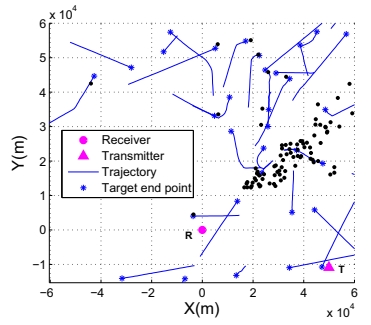

Figure: TNO measurements and ground truth 


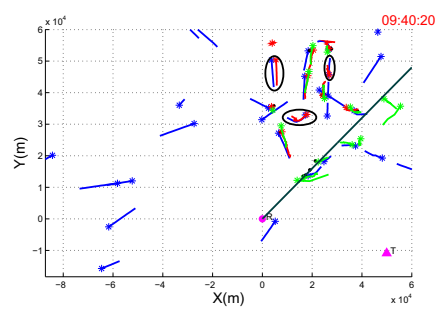

Figure: TNO and McMaster track comparison

- Blue - Truth; Red - McMaster tracks; Green - TNO tracks

- Formed more tracks than TNO tracker (marked by ellipses).

- Large number of false alarms are observed in a narrow region.

- Few targets in the coverage region did not get any measurement at all.

- Biases are observed in Doppler measurements for few tracks.

- Many false tracks are formed due to high false alarm rate. 
Real Data: HA/NATO Data (Multistatic)

McMaster

University

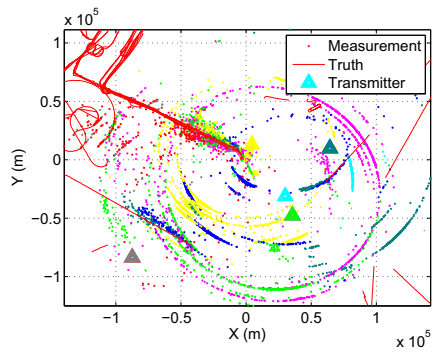

Figure: Ground truth and data from all transmitters at a threshold of $21 \mathrm{~dB}$

- Targets return measurements from only few transmitters.

Estimation, Tracking and Fusion Laboratory (ETFLab)

Analysis of HA Data

- Lower probability of detection makes track initiation difficult.

- Tracks are initialized by considering possible combinations of measurements from different transmitters.

- Initialized tracks with common measurements are merged.

- 2-D assignment is used to find association between tracks and measurements from each transmitter.

- Tracks are updated by augmenting the associated measurements from different transmitters. 


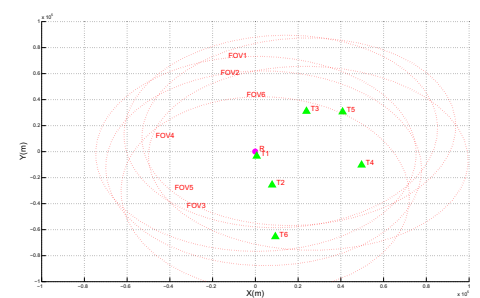

- Single receiver collects data from multiple transmitters operating with the same frequency.

- Measurements are bistatic range and bistatic doppler shift.

- Field of view (FOV) by only considering the maximum bistatic range.

\section{Analysis of CORA Data}

- Challenges:

- High false alarm rate

- No bearing information.

- Association between measurements and transmitters is unknown

- Multilateration is complicated by unknown transmitter association and high clutter.

- Simple track initiation methods cannot be used.

(1) Two-level tracking

- Range-Doppler tracking for clutter rejection.

- Track-to-track fusion with multilateration.

(2) Fusion with TNO data, which has less uncertainty

- Use TNO data to initiate and track the tracks.

- Use CORA data for track maintenance. 
Simulation (Multistatic)

McMaster

- Few errors are observed in the real data

- Simulation with exact configuration is considered here.

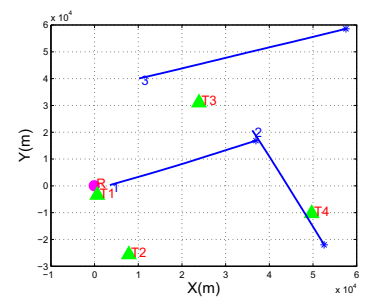

- There are 4 transmitters and one receiver.

- Measurement variance $\sigma_{r}^{2}=400 \mathrm{~m}^{2}$ and $\sigma_{\dot{r}}^{2}=100 \mathrm{~m}^{2}$.

Estimation, Tracking and Fusion Laboratory (ETFLab)

Simulation Results

McMaster

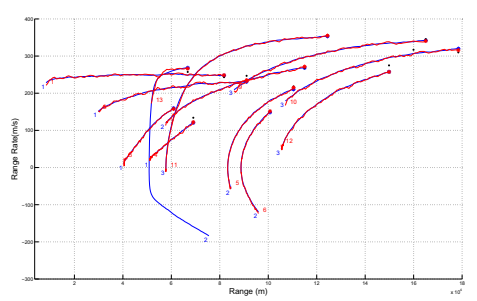

Figure: Tracks formed by assuming measurements are originated from closest transmitter

- Multiple tracks formed for each target.

- Track-to-track association needs to be done.

Estimation, Tracking and Fusion Laboratory (ETFLab) 
Resolving Transmitter Origin Uncertainty

McMaster

- Approach 1 :

- Track the targets in state space.

- Associate the measurements to targets and transmitters.

- Approach 2:

- Track the targets in measurement space assuming measurements are originated from closest transmitter.

- Multiple tracks can be formed for each target.

- Fuse the tracks by associating the tracks to transmitters.

- Convert tracks to state space.

- In both approaches, an additional assignment compared to the conventional radar system is required.

Approach 1: Tracking in State Space

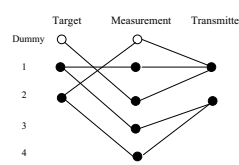

- $a(k, m, t, j)=1$, if $m$-th measurement is assigned to track $t$ and transmitter $j$.

- Objective is to minimize cost

- Subject to

$$
C(k \mid \mathbf{a}(k))=\sum_{m=0}^{M} \sum_{t=0}^{T} \sum_{j=1}^{N} a(k, m, t, j) c(k, m, t, j)
$$

$$
\sum_{m=0}^{M} a(k, m, t, j)=1, \quad t=1,2, \ldots, T \& j=1,2, \ldots, N
$$

$\sum_{t=0}^{T} \sum_{j=1}^{N} a(k, m, t, j)=1, \quad m=1,2, \ldots, M$

Estimation, Tracking and Fusion Laboratory (ETFLab) 
Approach 1: Tracking in State Space (cont'd)

- Dummy target (false alarm) and dummy measurement (miss detection) exist, but there is no dummy transmitter.

- Result in a suboptimal solution if $c(k, m, t, j)$ are calculated independently.

- New targets must be handled with different association procedure.

- New assignment technique:

- Each measurement is added to each transmitter

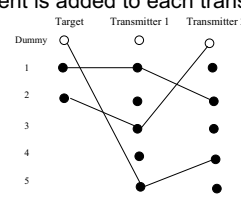

Figure: Assignment tree

- $i$-th measurement at each transmitter represents the same measurement.

- $i$-th measurement can be originated form only one transmitter. - A branch contains more than one $i$-th measurement is invalid

- Can be imposed by setting the cost of that branch to infinity.

- $i$-th measurement from any of the transmitter can be used with at most one target.

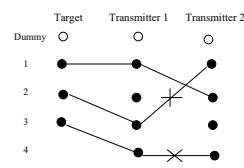

Figure: Infeasible assignments

- Solution to above assignment problem with all constraints under development. 
- Bias: It cannot be ignored.

- Imprecise tranmitter/receiver location: Need to incorporate into tracking/fusion.

- Non-uninform clutter: The real-world is not uniform.

- Non-stationary clutter parameters: Need to estimate clutter maps online (temporally and spatially).

- Bias with multiple sensors: More problematic.

- Fusion with different clutter models: Becomes challenging with sensors of different qualities.

\section{More Real-World Issues}

- Fusion: Fusion of outputs from MHT, Particle filters, PHD filters (very challenging)

- Fusion of intermittent data: Value of intermittent reports from accurate sensors (e.g., fusion of infrequent AIS data with OTH data)

- Out-of-sequence data: Incorporation of out-of-sequence data from disparate sensors

- Communication issues: 